\title{
The characterization of radioactive waste: a critical review of techniques implemented or under development at CEA, France
}

\author{
Bertrand Pérot ${ }^{1, *}$, Fanny Jallu ${ }^{1}$, Christian Passard ${ }^{1}$, Olivier Gueton ${ }^{1}$, Pierre-Guy Allinei ${ }^{1}$, Laurent Loubet ${ }^{1}$, \\ Nicolas Estre ${ }^{1}$, Eric Simon ${ }^{1}$, Cédric Carasco ${ }^{1}$, Christophe Roure ${ }^{1}$, Lionel Boucher ${ }^{1}$, Hervé Lamotte ${ }^{1}$, \\ Jérôme Comte ${ }^{1}$, Maïté Bertaux ${ }^{1}$, Abdallah Lyoussi $^{1}$, Pascal Fichet ${ }^{2}$, and Frédérick Carrel ${ }^{3}$ \\ ${ }^{1}$ CEA, DEN, Cadarache, 13108 Saint-Paul-lez-Durance, France \\ 2 CEA, DEN, Saclay, 91191 Gif-sur-Yvette, France \\ ${ }^{3}$ CEA, LIST, Saclay, 91191 Gif-sur-Yvette, France
}

Received: 11 July 2017 / Received in final form: 30 October 2017 / Accepted: 23 November 2017

\begin{abstract}
This review paper describes the destructive and non-destructive measurements implemented or under development at CEA, in view to perform the most complete radioactive waste characterization. First, high-energy photon imaging (radiography, tomography) brings essential information on the waste packages, such as density, position and shape of the waste inside the container and in the possible binder, quality of coating and blocking matrices, presence of internal shields or structures, presence of cracks, voids, or other defects in the container or in the matrix, liquids or other forbidden materials, etc. Radiological assessment is then performed using a series of non-destructive techniques such as gamma-ray spectroscopy, which allows characterizing a wide range of radioactive and nuclear materials, passive neutron coincidence counting and active neutron interrogation with the differential die-away technique, or active photon interrogation with high-energy photons (photofission), to measure nuclear materials. Prompt gamma neutron activation analysis (PGNAA) can also be employed to detect toxic chemicals or elements which can greatly influence the above measurements, such as neutron moderators or absorbers. Digital auto-radiography can also be used to detect alpha and beta contaminated waste. These nondestructive assessments can be completed by gas measurements, to quantify the radioactive and radiolysis gas releases, and by destructive examinations such as coring homogeneous waste packages or cutting the heterogeneous ones, in view to perform visual examination and a series of physical, chemical, and radiochemical analyses on samples. These last allow for instance to check the mechanical and containment properties of the package envelop, or of the waste binder, to measure toxic chemicals, to assess the activity of long-lived radionuclides or pure beta emitters, to determine the isotopic composition of nuclear materials, etc.
\end{abstract}

\section{Introduction}

Safety in radioactive waste treatment, processing, interim and long-term storage facilities, retrieval of old waste, the regulations on transport of radioactive materials and the French law on transparency and nuclear safety require a thorough characterization of radioactive waste at different stages. In this context, the CEA implements or develops, with the support of major partners such as ANDRA and AREVA, a whole range of both non-destructive and destructive measurement methods allowing access to the physical (density, volume, shape, position of the waste and embedding matrixes, quality control, mechanical toughness, cracking, diffusion coefficient, gas release, thermal power, etc.), chemical (elemental composition, content of toxic or reactive substances, etc.) and radiological

\footnotetext{
* e-mail: bertrand.perot@cea.fr
}

characteristics (dose rate, $\alpha$ and $\beta$ activity, isotopic composition and mass of nuclear materials, etc.) of nuclear waste or nuclear waste packages. The complementarity of the measurement methods is effectively demonstrated by the Super-COntrôles which are second-level examinations (first level examinations are the responsibility of the waste producers) carried out at the request of ANDRA (The French national agency for management of radioactive waste) on certain waste packages intended for surface repository. Their purpose is to verify that they comply with the acceptance criteria of the aube storage centre and the approval documents issued by ANDRA for waste package producers. Many overviews of worldwide radioactive waste characterisation approaches already exist, such as [1] and references therein, and several reference books describe the detailed principle of nuclear measurement techniques, such as [2-4]. The aim of this paper is to focus on techniques and equipment implemented or under development at CEA. 


\section{The passive non-destructive measurements}

\subsection{Introduction}

The methods termed passive consist in measuring the radiation emitted spontaneously by the radioactive materials. In addition to simple dose rate measurements, gamma spectrometry is the most widespread technique because it is relatively simple and inexpensive to use and can identify and quantify gamma-emitting radionuclides as well as determine the isotopic composition of nuclear materials. Nevertheless, it is sometimes necessary to implement neutron counting, particularly in characterizing nuclear materials whose gamma emission is masked by that of more intense emitters, such as fission or activation products, or for the purpose of obtaining complementary information and reducing measurement uncertainties, such as those linked to the attenuation of gamma and neutron radiation in packages. In this context, we may mention the emergence of calorimetry (not described in this paper) in the field of package characterization of alpha or tritiated waste, because this technique is insensitive to these attenuation effects. At last, autoradiography is a technique for revealing fixed radioactivity that is difficult to measure in certain types of waste, using sensitive screens, especially beta emitters such as tritium, and in some instances alpha emitters.

\subsection{Gamma spectrometry}

Some radionuclides emit characteristic X-ray and gamma radiation with specific energies and intensities, which makes it possible to identify them and even quantify them, by non-destructive measurement.

The general principle of gamma spectrometry consists in measuring these photons by a sensor which delivers a signal proportional to the energy, which is then analysed and classified in the form of a histogram called a gamma spectrum (Fig. 1).

Two phenomena based on the ionization of the sensor material are used to detect X-ray and gamma photons: - either the ionization generates charges in a nonconductive material which are then transformed into electrical pulses or current by means of an electrical polarization, this signal being amplified afterwards by specialized electronics (gas detectors, semiconductors); - or the primary ionization is transformed into light photons (scintillators) which are then collected and amplified by a photomultiplier or a photodiode.

Ideally, when a photon deposits all its energy in the detector, a very narrow peak (intrinsic width related to the lifetime of the excited state) should appear on the spectrum. However in practice, this peak is broadened due to the statistical fluctuations in the detection process and the noise added by the processing electronics. This widening, called energy resolution, mainly depends on the detector and reflects its ability to separate different gamma emitting isotopes at neighbouring energies.

Another parameter to be taken into account when choosing the material for quantitative measurements is detection efficiency, which makes it possible to connect the

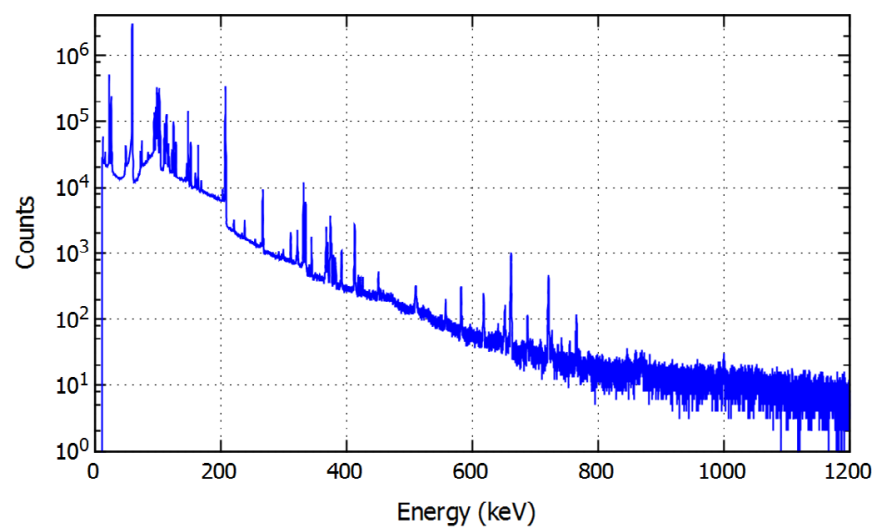

Fig. 1. Gamma spectrum obtained with a hyper-pure germanium (HPGe) detector in which each peak corresponds to a gamma emission line of the source.

surface of the observed peak on the spectrum with the corresponding activity of the radioelement. This characteristic depends on the energy of gamma radiation, its attenuation in the measured object, the type of detector selected (material, density) and its useful volume. The determination of the efficiency is carried out by means of radioactive standards having the same size and composition of the object to be measured, or numerically with computer codes simulating the transport of X-ray and gamma photons, two approaches now being used more and more in a complementary manner.

The main gamma spectrometry detectors are:

- inorganic (NaI, CsI, LaBr, BGO) and organic (plastic or liquid) scintillators. The latter can be doped with lead or another material with a high atomic number to improve their sensitivity to gamma radiation;

- hyper-pure germanium (HPGe), silicon, CdTe or CdZnTe, GaAs semiconductors.

Their main characteristics can be summarized as follows:

- scintillators can be manufactured in large volumes (notably $\mathrm{NaI}$ and plastics) but generally have poor energy resolution. They are therefore often used for low intensity photon flux measurements with simple gamma spectra. However, since most scintillators are very fast (signals of the order of nanoseconds), they can also be used at high counting rates or for coincidence counting; - semiconductors operating at room temperature (mainly CdTe and CdZnTe) have crystals of very small size (of the order of $\mathrm{mm}^{3}$ to $\mathrm{cm}^{3}$ ) which enables them to withstand intense photon fields. The spectral resolution is slightly better than that of scintillators;

- HPGe are characterised by an excellent energy resolution, which makes it possible for them to distinguish the numerous gamma and X-ray lines emitted by the nuclear material (uranium, plutonium, etc. see Fig. 2, left box) and to deduce their isotopic composition (see Fig. 2, right box). They constitute the reference in gamma spectrometry, with volumes ranging from $\mathrm{cm}^{3}$ to about 1 litre and several types of refrigeration (liquid nitrogen, cryogenic compressor, combination of both) allowing their use over a wide range of applications. 


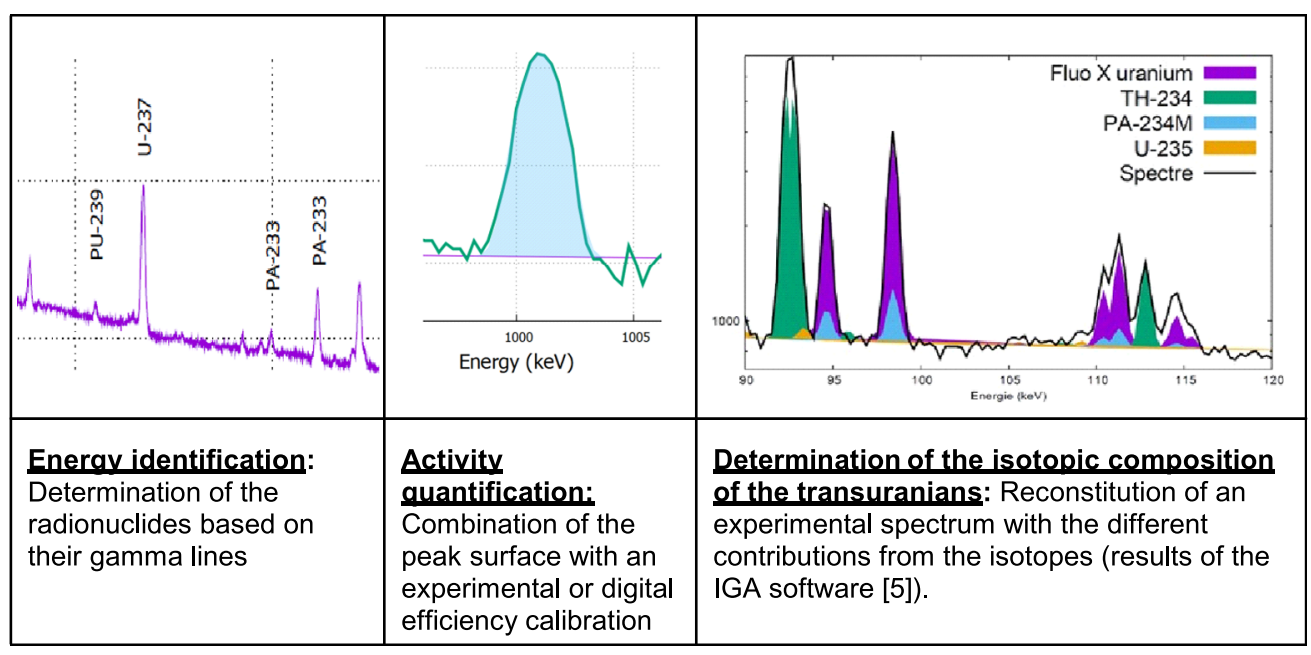

Fig. 2. Main uses of an HPGe gamma spectrum (right panel taken from IGA software [5]).

In general, the measuring station is made up of the detector, an analogue or digital electronics system and an analysis system. Depending on the need, this assembly can be integrated into a system allowing remote control in the positioning of the detector, the rotation of the object to be measured, the placement of collimators or screens adapted to the measurement configuration.

\subsubsection{Application $\mathrm{n}^{\circ} 1$ : the global measurement of waste containers}

Gamma spectrometry is frequently used to measure waste packages because it can meet the characterization requirements for a wide range of very different types of containers (polyethylene bins of a few litres, metal drums ranging from $100 \mathrm{~L}$ to $1 \mathrm{~m}^{3}$, concrete packages, etc.), the physicochemical nature, density, volume, activity level, isotopic spectrum and localization of radioelements.

However, this measurement technique may prove to be limited by the attenuation of the radiation in the material. For very dense materials (eg: concrete of a $2-3 \mathrm{~g} \cdot \mathrm{cm}^{-3}$ density), the measurable depth of matrix is only a few centimetres for the gamma emissions of the major radioactive isotopes ( $50 \mathrm{keV}$ to $2 \mathrm{MeV}$ ). In some instance, this constraint force concrete waste to be broken up and measured in small baskets before it is placed in a large volume container (between 1 and $10 \mathrm{~m}^{3}$ ).

The gamma-ray spectrometry station of Figure 3 consists of two measurement channels: (1) a low-energy (typically below $1 \mathrm{MeV}$ ) planar HPGe detector with an entrance surface area of $3700 \mathrm{~mm}^{2}$, which is mainly used to determine the isotopic composition of plutonium and uranium; (2) a wide energy $[0-4 \mathrm{MeV}]$ coaxial HPGe detector of $40 \%$ relative efficiency, for the identification and quantification of measurable radionuclides. For each channel, the detector height and the distance between the germanium crystal and the waste package are mechanically adjusted according to the physical characteristics of the package determined by high-energy photon imaging (height, dimension, and position of the waste within the matrix) and of its contact dose rate previously measured.

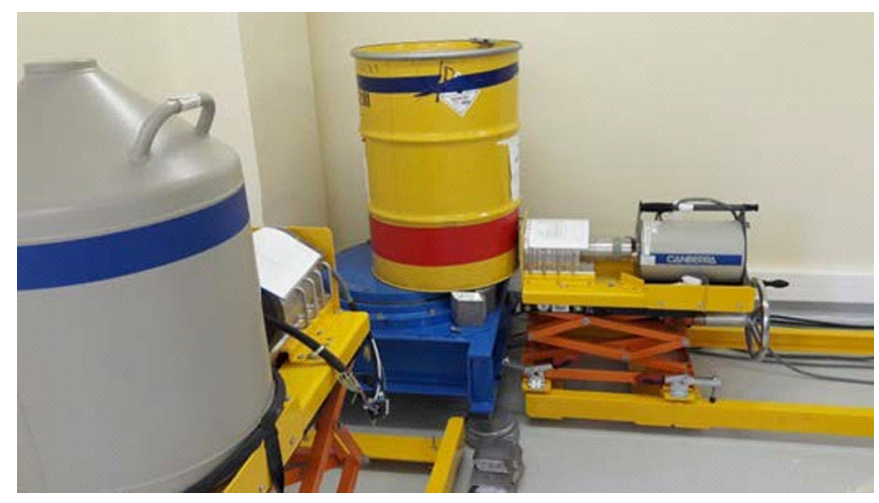

Fig. 3. Gamma spectroscopy station for 100 to $200 \mathrm{~L}$ waste drums, in CHICADE basic nuclear facility, at CEA Cadarache.

\subsubsection{Application $\mathrm{n}^{\circ}$ 2: Segmented measurements and emission tomography}

To perfect determination of the activity contained in waste containers, the overall measurement of the package can be replaced by a series of focused measurements using a collimator reducing the field of view of the detector, which scans the package by means of a system of movement (translation, rotation, elevation) of the package, the detector or a combination of both. We distinguish:

- gamma scanning measurements in which the collimator has an opening angle that allows the measurement of a complete slice in the width of the package which is scanned vertically;

- the emission tomography (see principle in Fig. 4) which relies on a collimation reduced to a segment of the package and requires a horizontal scan coupled with angular acquisitions, making it possible to reconstitute the spatial distribution of the activity in the tomographic section. This operation can be repeated in different sections to reconstruct the $3 \mathrm{D}$ activity of the waste. The tomographic reconstruction step requires knowledge of 


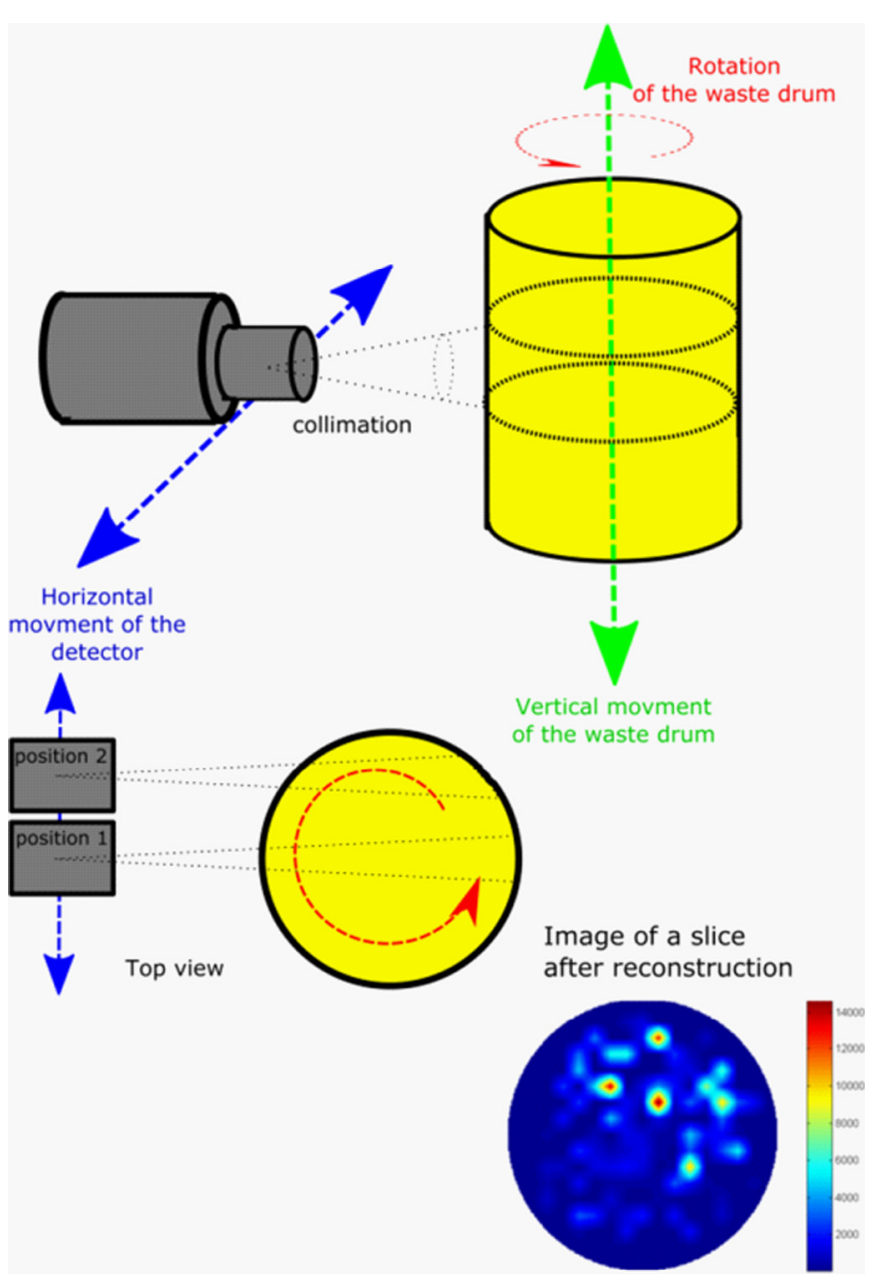

Fig. 4. Principle of gamma emission tomography.

the gamma attenuation by the materials in the waste container and therefore complementary photon transmission measurements.

\subsubsection{Application $\mathrm{n}^{\circ}$ 3: stationary waste measurement}

In the context of cleaning and dismantling nuclear facilities, some large components (like the vessel of Fig. 5, or steam generators, tank lids, compressors, etc.) must be characterised before they can be assigned to the appropriate waste disposal site. Gamma spectrometry makes it possible to determine the activity and the distribution of radio-tracers (measurable radioelements) in the appraised component. The activity of the other nonmeasurable $\alpha$ and $\beta$ emitting radioelements is obtained by means of ratios determined by:

- radio-chemical analyses carried out on samples;

- calculations of activation, radioactive decay, and if necessary transfer of the various radionuclides produced in reactor and in nuclear processes.

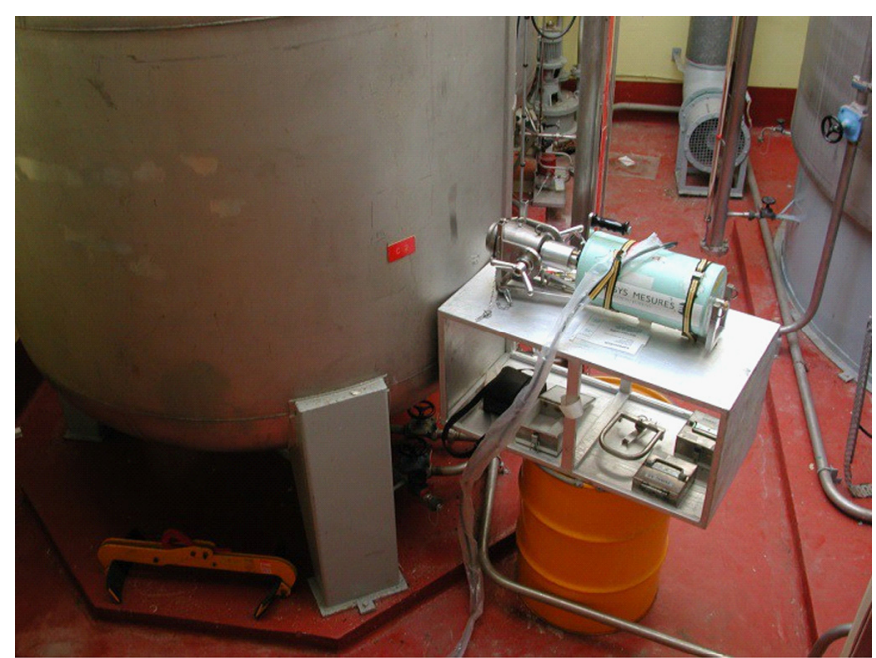

Fig. 5. Measurement of a vessel that contained contaminated effluents (HPGe detector).

\subsection{Passive neutron measurement}

Passive neutron measurement [4] is a non-intrusive characterisation method that provides information on the quantity of actinides present in an object, notably radioactive waste for this paper, but it is also more widely applied in many fields such as safeguards (controls of nuclear materials), or cleaning and dismantling nuclear facilities. It is mainly used for the quantification of plutonium, taking advantage of the spontaneous emission of neutrons which follows the disintegration of heavy nuclei and which arises mainly from two origins:

- spontaneous fission, accompanied by the emission of 2 to 4 fast neutrons on the average, particularly intense for isotopes 238, 240 and 242 of plutonium, 242 and 244 of curium, the main emission in radioactive waste arising from ${ }^{240} \mathrm{Pu}$ and ${ }^{244} \mathrm{Cm}$;

- the $(\alpha, n)$ reactions which produce only a single fast neutron following the interaction of the $\alpha$ particle emitted during the disintegration with a light element present in the medium (Be, B, C, O, F, etc.). They are particularly intense for actinides with a short $\alpha$ radioactive period $\left({ }^{238} \mathrm{Pu},{ }^{241} \mathrm{Am}\right.$, etc. $)$.

The main advantage of this method is its relatively low sensitivity to the density of materials surrounding the nuclear material, such as, for example, the matrix in a waste package. However, it is impacted by the presence of hydrogen and other light elements that slow down neutrons, making some of them undetectable. In contrast, it is not very sensitive to metallic waste matrices compared to gamma spectrometry, frequently used in radioactive waste measurements, making these two techniques quite complementary.

In its simplest mode of application, the passive neutron measurement aims to detect all neutrons, without distinction of emission process, thanks to sensors positioned near 


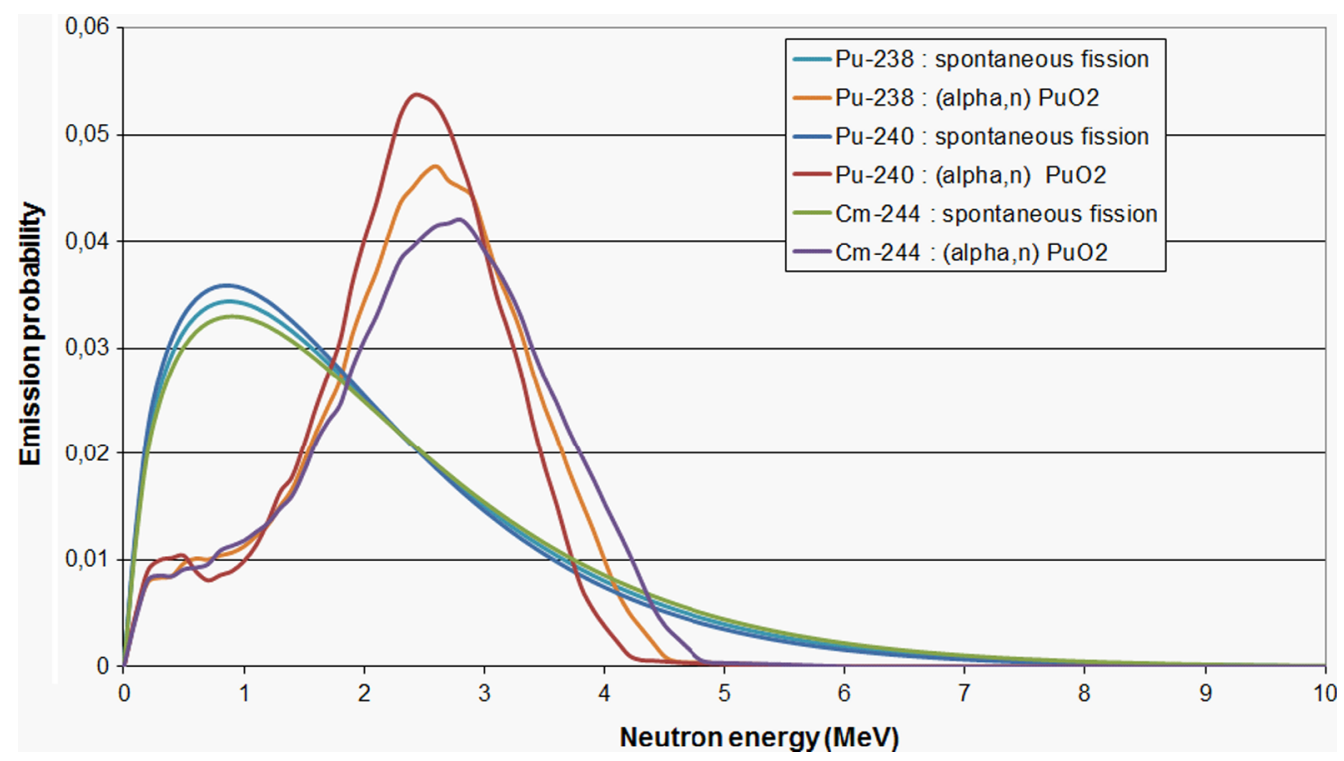

Fig. 6. Neutron emission energy spectra of different isotopes by spontaneous fission and by $(\alpha, n)$ reaction on oxygen (actinide oxides).

the object to be characterised: it is the total neutron counting. Depending on the application, ${ }^{3} \mathrm{He}$ or boron coated proportional counters will be used, or fission chambers, liquid or plastic scintillators, etc. However, this approach has the disadvantage, particularly in the field of radioactive waste, of having a high sensitivity to the chemical form of the contaminant (metallic, oxide, or fluorinated plutonium, americium, or other actinides) via its $(\alpha, n)$ component for which neutron production can vary by a factor of 1000 with the nature of the light element.

To compensate for this disadvantage, it is necessary to discriminate the signal fraction originating from the spontaneous fissions from that resulting from $(\alpha, n)$ reactions, thanks to the difference existing in the number of neutrons emitted per reaction. The use of a time correlation analysis of the signals can determine the number of neutron pairs emitted by the contaminant (classical neutron coincidence counting) or even the number of higher order coincidences, such as triplets (counting of neutron multiplicities). Since the $(\alpha, n)$ reaction produces only one neutron, coincidences can only come from fission, thereby providing information independent of the chemical form.

Unlike measurements by gamma spectrometry, it is not possible to identify precisely the emitting isotope by knowing the energy of the detected neutron, since neutrons are produced according to a continuous spectrum, whatever the actinide, with mean energies close to $2 \mathrm{MeV}$ in spontaneous fissions and in $(\alpha, \mathrm{n})$ reactions with oxides (see Fig. 6).

Precise interpretation of the results therefore requires knowledge of the isotopic composition of the contaminant, either by the traceability of the object (reference spectrum) or by a specific gamma spectrometry measurement. Failing this, only an overall assessment representing all the potential emitting isotopes will be available.
The presence of curium may also turn out to be limiting, due to its very high neutron emission, when the objective is the quantification of plutonium, masking that of the latter.

Although less widespread than gamma spectrometry for the characterization of radioactive waste, passive neutron measurement, particularly in its variant of coincidence counting, is still in common use.

The device in Figure 7 illustrates the concept of a passive neutron measuring station based on total counting for the characterisation of large compressors removed from the fuel enrichment plant at Pierrelatte, France, during dismantling operations [6].

For this potentially contaminated uranium waste of a known fluorinated chemical form, which is favourable to $(\alpha, n)$ emission, the objective is to ensure that the ${ }^{235} \mathrm{U}$ quantity remains below the transport threshold of $15 \mathrm{~g}$. After a design based on numerical simulation along with a calibration phase, also numerical, the measuring station, consisting of polyethylene walls in which $44{ }^{3} \mathrm{He}$ proportional counters were inserted, allowed the monitoring and subsequent transport of about a thousand compressors, with a detection limit around $5 \mathrm{~g}$ of ${ }^{235} \mathrm{U}$ in $15 \mathrm{~min}$ of measurement. This measurement demonstrated that almost all compressors met the transport threshold.

The passive neutron measuring station in Figure 8 presents a typical example of a neutron coincidence counting device [7]. The objective is to characterize the mass of plutonium present in several thousand $100 \mathrm{~L}$ drums containing technological waste such as metal, glass, plastic or cellulose with a detection limit close to $1 \mathrm{~g}$ of plutonium. In order to achieve these performances, two other non-destructive nuclear measurement methods have been combined:

- an X-ray imaging station providing a spatial resolution of $1 \mathrm{~mm}$, making it possible to obtain the filling height of the drum as well as a partial indication of the nature of the waste; 

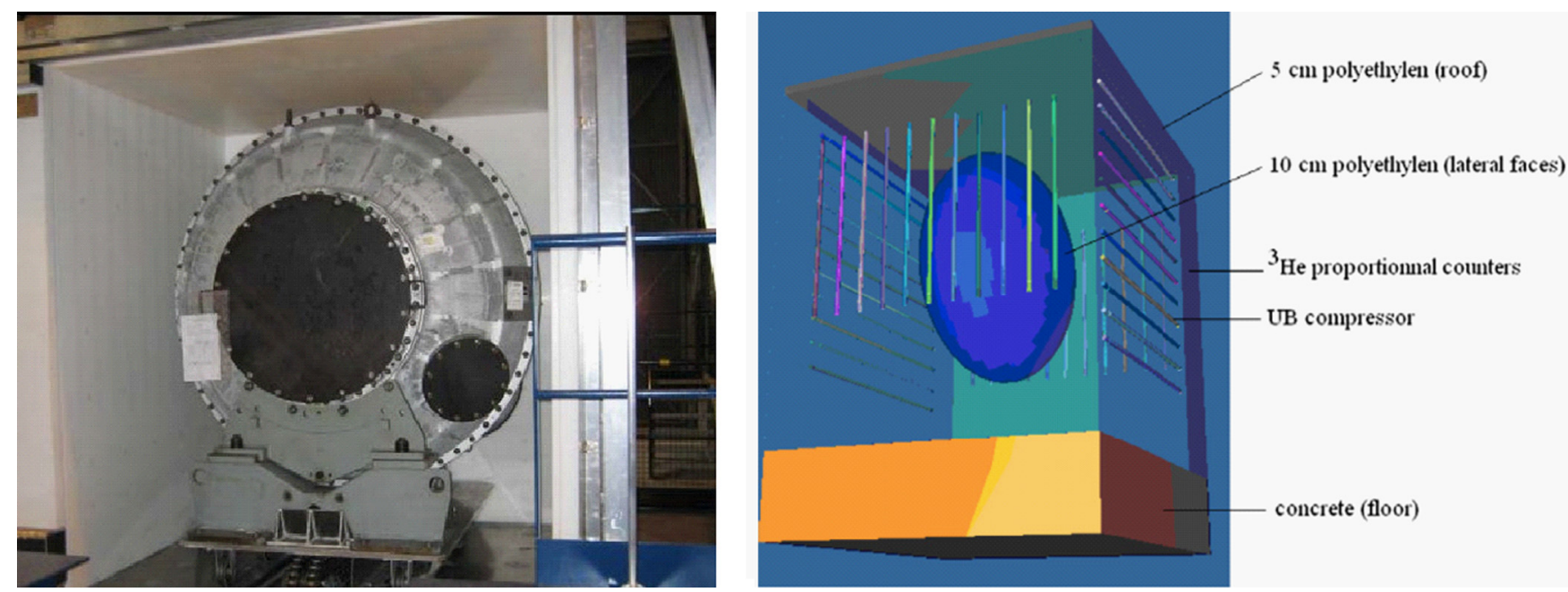

Fig. 7. Photograph of a compressor in the MECANO measuring station (left) and MCNP simulation diagram (right).
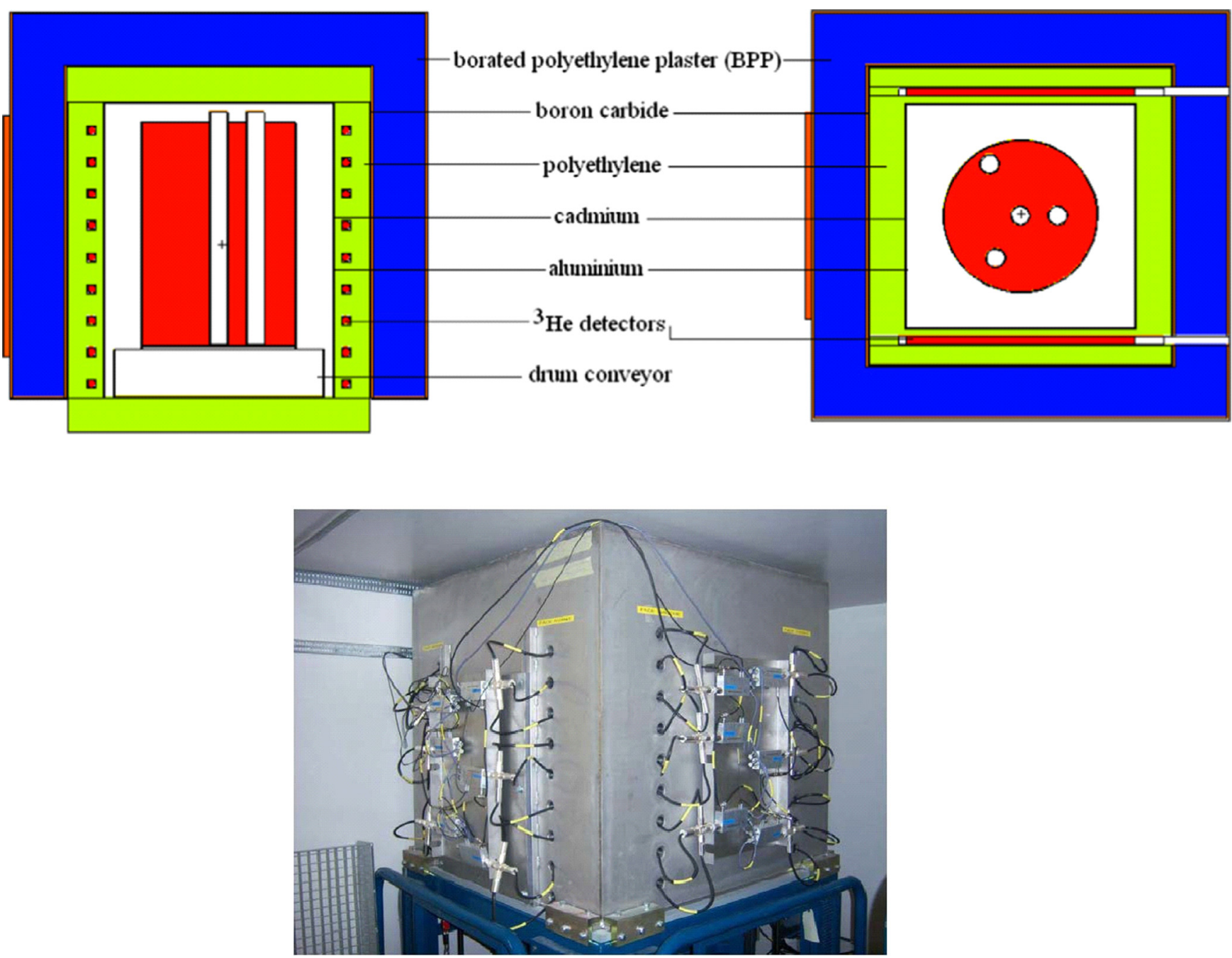

Fig. 8. Photograph of the neutron measurement station at the PEGASE facility in CEA Cadarache and simulation model with a Monte Carlo neutron-gamma transport code, showing a calibration drum filled with inactive materials representative of the waste matrix and hollow tubes to introduce radioactive sources $\left({ }^{252} \mathrm{Cf}, \mathrm{AmBe}\right)$ or $\mathrm{Pu}$ samples (calibration standards). 

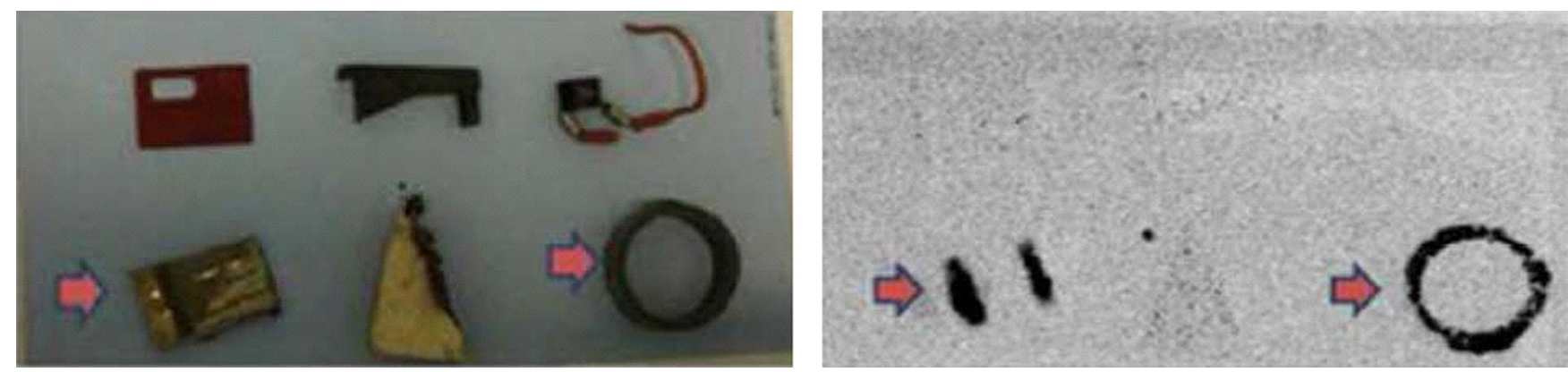

Fig. 9. Measurement of tritiated waste by digital autoradiography.

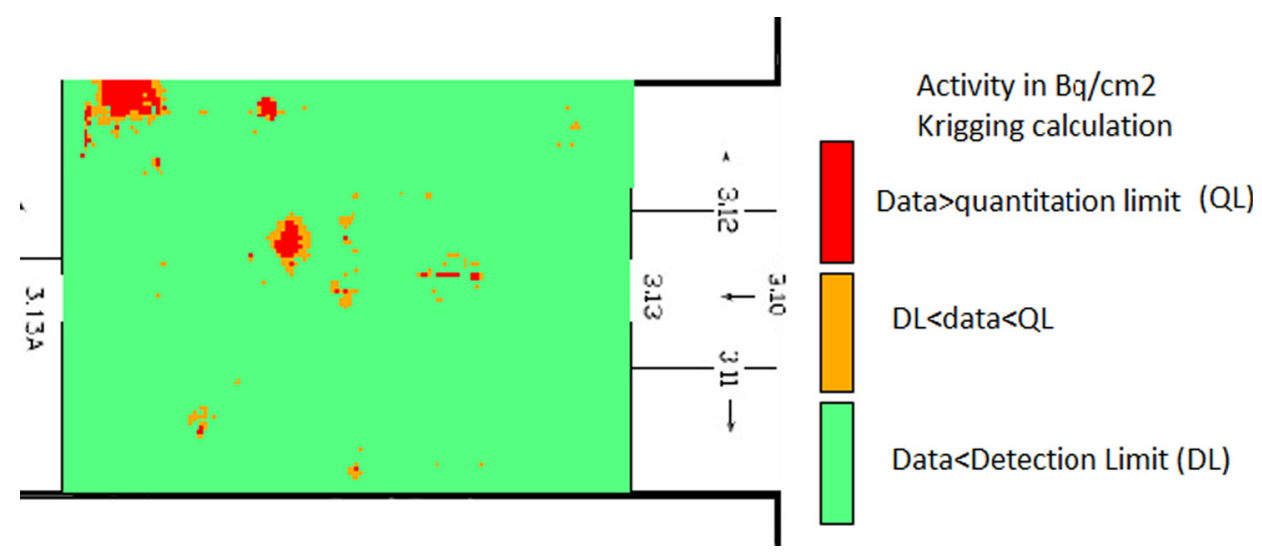

Fig. 10. Tritium mapping at the surface of a $150 \mathrm{~m}^{2}$ laboratory after geostatistical calculation.

- a gamma spectrometry station designed to obtain the activities of the ${ }^{235} \mathrm{U},{ }^{238} \mathrm{U}$ and ${ }^{239} \mathrm{Pu}$ isotopes when they are measurable, as well as the isotopic composition of plutonium necessary for the accurate interpretation of the neutron coincidence measurement.

The neutron measurement station consists mainly of polyethylene walls in which $36{ }^{3} \mathrm{He}$ proportional counters were inserted, a neutron screen made of cadmium bordering the inner walls of the measurement cavity to reduce neutron lifetime by absorbing thermal neutrons, and a boron carbide neutron screen as well as an external boron polyethylene plaster protection to reduce the background from the outside. Its design has been optimized by numerical simulation and an experimental calibration covering the different types of waste allowed qualifying its performances, in particular in terms of uncertainty associated with the measured plutonium mass, which has a relative standard deviation of $35 \%$. The electronics based on shift registers analyses time correlation of the signals coming from the detectors.

\subsection{Digital autoradiography for beta and alpha measurements}

The digital autoradiography used for several decades for applications in biology and geology, is a non-destructive nuclear analysis method that has proved applicable on solid materials (metals, powder, smears, concrete, wood, etc.) resulting from dismantling operations. The first operational developments on actual dismantling worksites imple- mented autoradiography screens reusable and sensitive to all types of radiation [8,9]. The non-intrusive detection of radionuclides that are difficult to measure (low-energy $\beta$ emitters such as ${ }^{3} \mathrm{H}$ and ${ }^{14} \mathrm{C}$, and alpha emitters) has thus become possible with, in particular, improved sampling of low-tritiated waste. The autoradiography can produce and quantify an image of the radioactivity present in a sample. Waste with a potentially fixed radioactivity is deposited on the screens; after a certain exposure time, these screens are developed to obtain an image revealing the presence of the radioisotopes (the two waste items marked with a red arrow in Fig. 9).

The imagery resulting from this technique also made it possible to make maps of traces of tritium, carbon 14 and uranium on civil engineering concrete (Fig. 10). Numerous imagery data has been shown to be compatible with the geographic information system and geostatistical calculations.

Current developments aim at optimizing the technique for dismantling operations by making it possible to move from offline detection to detection in real time.

\subsection{Analysis of the gases}

The degassing measurement of the waste packages (or a block of waste) has two main objectives, first the measurement of the radioactive gases released by the package, and second the analysis of the radiolysis gases produced by the package. A number of radioactive isotopes are found in gaseous compounds that are likely to escape from packages. 


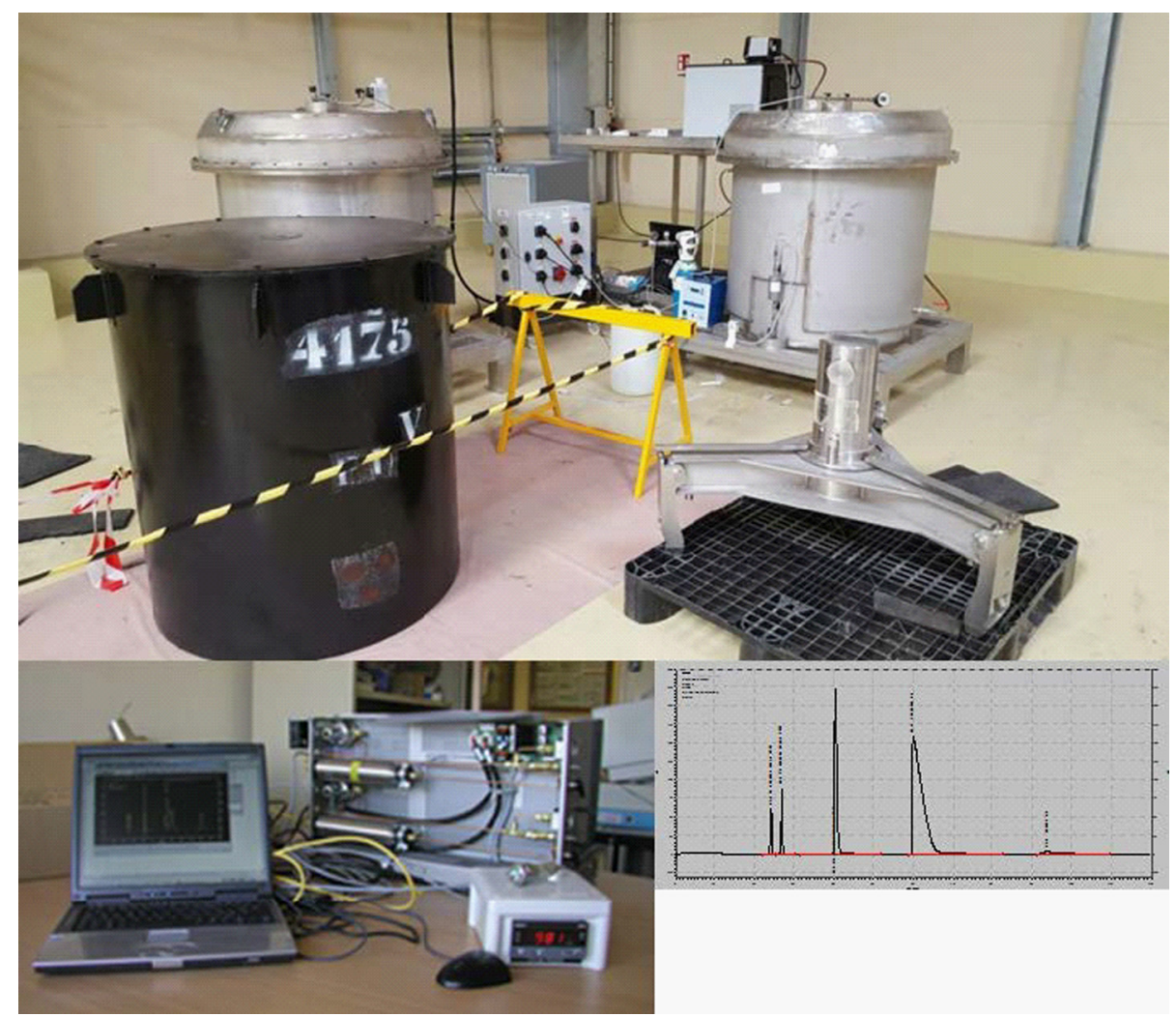

Fig. 11. Hydrogen degassing measurement station on $870 \mathrm{~L}$ packages (in black) at the CEDRA storage facility, CEA Cadarache. The containment enclosures (grey), adapted to the type of package, allow the accumulation of gases released from the package. A sample of the atmosphere of the chamber is then measured by gas chromatography (bottom left). The chromatogram (bottom right) shows the separation of the different gases in the atmosphere (hydrogen corresponds to the second peak).

Such is the case of tritium, carbon 14, chlorine 36 or radon 226. Similarly, certain constituents of the waste or container (plastics, concrete, water or bitumen) are likely to produce gases under the effect of irradiation. These gases, in particular hydrogen, may represent a hazard in storage and therefore must be monitored [10]. The measurement technique consists in placing the waste package in a sealed enclosure (Fig. 11), allowing the gas to accumulate in this chamber and then measuring the quantity of gas (by gas chromatography for the radiolysis gases, or by trapping and scintillation counting for radioactive gases such as ${ }^{3} \mathrm{H}$ or ${ }^{14} \mathrm{C}$ ) in order to deduce the release rate. If this measurement is associated with the source term of the package (quantity of plastic, for example, and irradiation capacity), it is possible to model the radiolysis gas production over time and to estimate the quantities released in a storage unit in the duration. Similarly, by knowing the source term of the radioactive isotopes present in the package, the degassing measurement makes it possible to deduce a rate of release and therefore the impact of the package over time.

\section{Active non-destructive measurements}

\subsection{Introduction}

These techniques use an external source of radiation and are inherently more complex to implement than the previous passive methods. High-energy photon imaging provides very rich information about the internal structure of packages (density, shape, position, etc. of the waste, coating and blocking matrices, internal screens, containers, etc.). Active neutron interrogation consists in measuring the fast and delayed neutrons of neutron-induced fissions and allows it to quantify the fissile materials, for example when passive neutron measurement is inoperative because their spontaneous neutron emission is insufficient or is masked by that of more intense emitters such as curium and americium. Highenergy photon interrogation (detection of photofission delayed neutrons or gamma rays) is studied to characterise the same nuclear materials in large-volume concrete packages for which neutron interrogation is limited due to strong neutron attenuation by hydrogen nuclei. Finally, neutron activation analysis (radiative capture prompt gamma radiations or fission delayed gamma rays) is studied to characterise chemical toxics or nuclear materials.

\subsection{High energy photon imaging}

In order to inspect the interior of a waste package without having to carry out a destructive action, high-energy photon imaging technique (radiography and tomography) can be used in the manner of a medical scanner. The package to be inspected is placed between the photon source and the detector. The image delivered by the detector reports the absorption of photons through the object. The thicker and denser the object, the greater the absorption. 


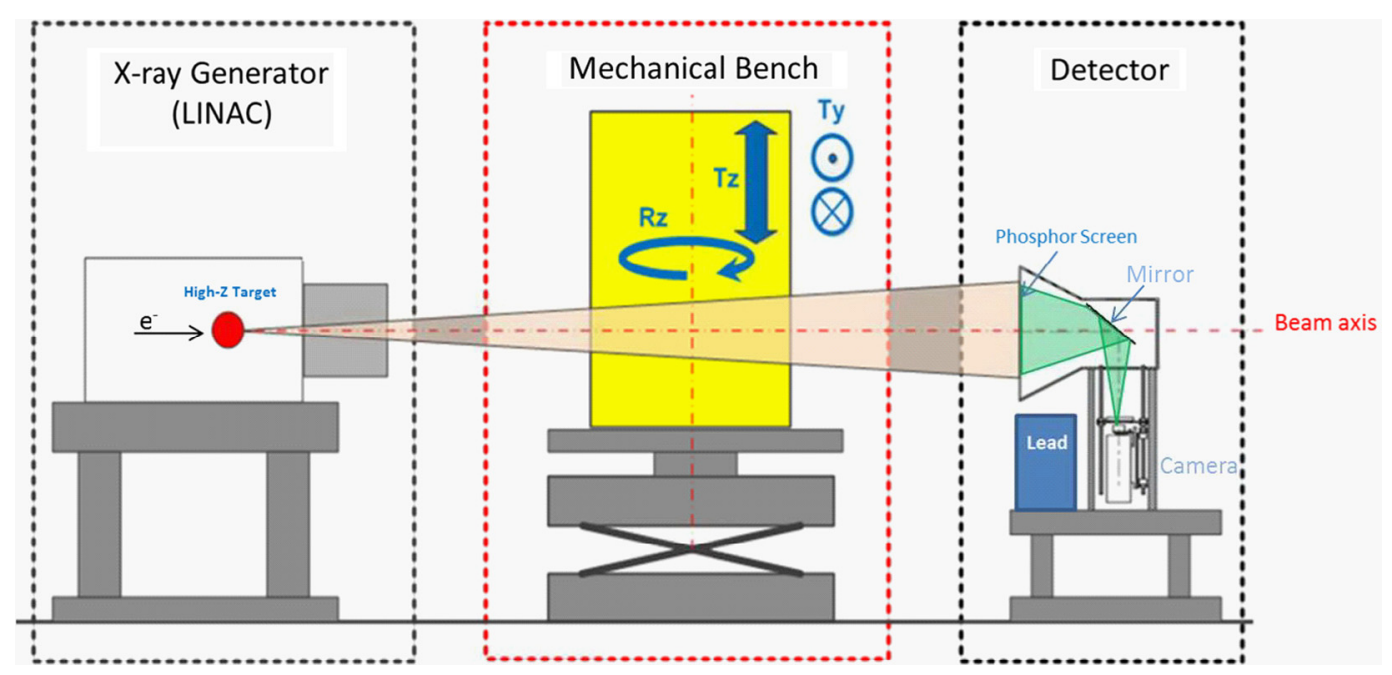

Fig. 12. Main components of the high-energy imaging system.

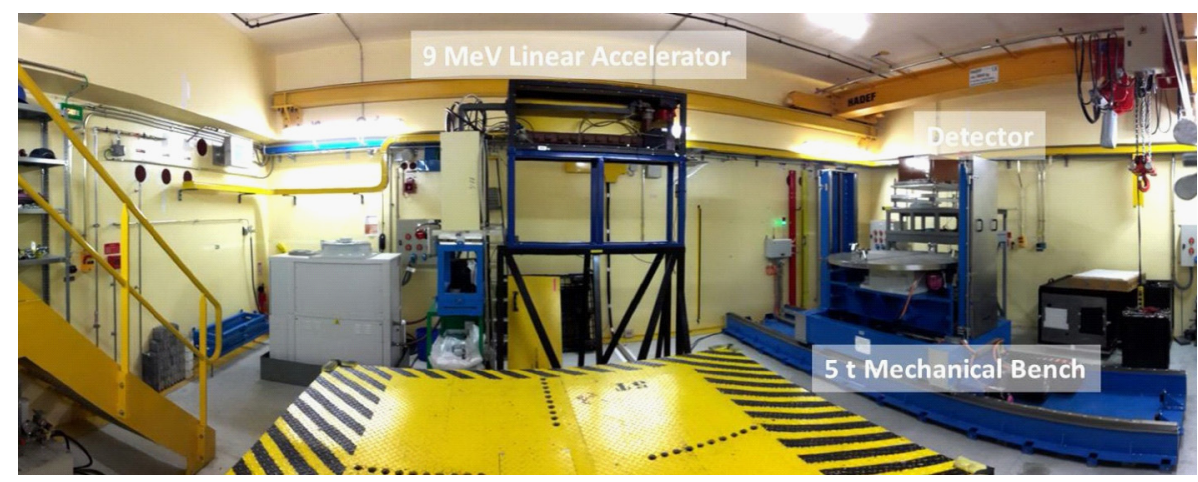

Fig. 13. The CINPHONIE cell hosting a high energy photon imaging system (the $9 \mathrm{MeV}$ LINAC will be replaced soon by a $20 \mathrm{MeV}$ LINAC), in the CHICADE basic nuclear facility, at CEA Cadarache.

However the analogy with medical scanners has its limits because they are sized to inspect a human body, a thickness of a few tens of centimetres of water, while a package of waste may be of variable size and density: ranging from a diameter of $60 \mathrm{~cm}$ and not very dense (less than $0.5 \mathrm{~g} \cdot \mathrm{cm}^{-3}$ ) for a drum of $220 \mathrm{~L}$ of bulk technological waste, to packages of more than $100 \mathrm{~cm}$ in diameter containing concrete and steel, such as the $2 \mathrm{~m}^{3}$ drums (with a density higher than $2 \mathrm{~g} \cdot \mathrm{cm}^{-3}$ ).

To grasp such objects, a high intensity and high energy photon source is required, such as that which can be produced by an electron linear accelerator (LINAC). This type of apparatus is first of all made up of an electron canon that produces electron packets. The latter are then accelerated in a network of cavities by a stationary HF wave and their energy is raised up to a few $\mathrm{MeV}$. The electrons are eventually projected onto a target made of heavy material (tungsten or tantalum). They then yield a fraction of their energy by emitting a braking radiation, or Bremsstrahlung. The dose rates delivered by these machines are very high, ranging from 10 to $100 \mathrm{~Gy} \mathrm{~min}{ }^{-1}$ at a distance of $1 \mathrm{~m}$ from the target, in the axis of the beam.
The radiographic image provides a projection of the object of millimetre resolution, each pixel of the image being representative of the attenuation experienced by the beam along a linear path. The tomographic image is obtained by acquiring different angular projections (radiographies) of the package. The tomographic reconstruction is then carried out by dedicated algorithms allowing precise visualization of the interior of an object (values of the density) in planar sections. The principle of such a system is shown in Figure 12.

With these levels of intensity and energy, it is possible to cross through more than a meter of concrete while keeping a detectable signal behind the object to be radiographed. However, it is absolutely necessary to set up highly efficient biological protection in order to protect the personnel. In CEA Cadarache, such a system is implemented in the CINPHONIE buried casemate containing a $9 \mathrm{MeV}$ LINAC, see Figure 13 [11].

This high-energy photon imaging system allows the realization of X-ray radiographies (2D imaging) or tomographies (sectional reconstruction of the interior of the package, 3D imaging), see Figure 14. 


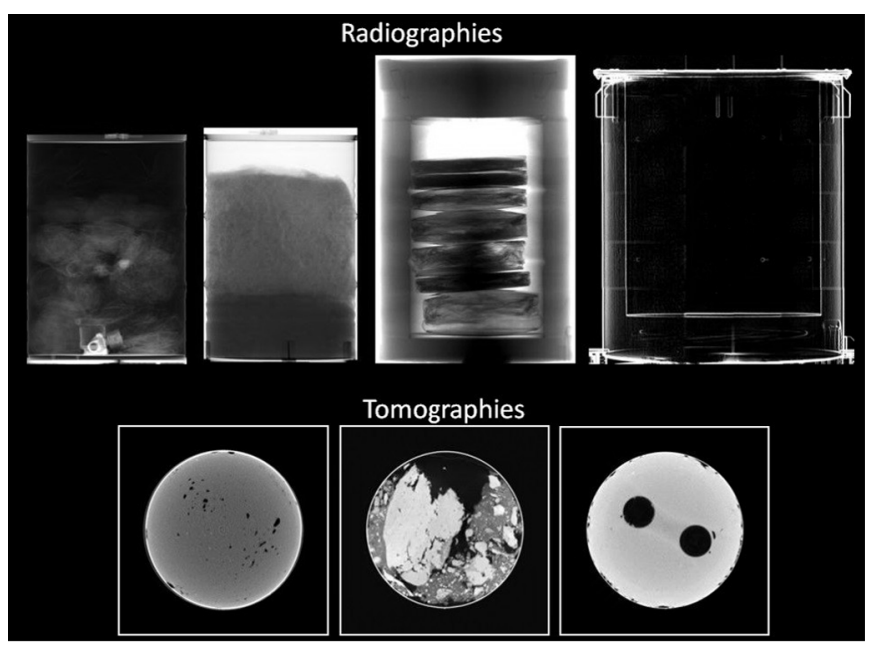

Fig. 14. Examples of X-ray radiographies and tomographies on packages of different diameters.

Thanks to standards of known and calibrated materials, it is finally possible to qualify the performances of the high energy $(9 \mathrm{MeV})$ tomograph of CINPHONIE: the spatial resolution is $1.5 \mathrm{~mm}$ and the precision on density is less than $10 \%$. Typical $1 \mathrm{~m}^{3}$ packages can be inspected in less than $10 \mathrm{~min}$ for a complete radiography, and about 30 min for a tomographic cut.

Two acquisition systems developed by CEA LETI are currently available: (1) a 2D wide-field screen of $80 \times 60 \mathrm{~cm}^{2}$ with a Gadox $\left(\mathrm{Gd}_{2} \mathrm{O}_{2} \mathrm{~S}\right)$ phosphor coating for rapid imaging, with attenuation dynamics of about 3 decades, or in other words $1 \mathrm{~m}$ of concrete or $25 \mathrm{~cm}$ of steel, and (2) a system of $25 \mathrm{CdTe}$ semiconductor bar detectors, with collimators oriented towards the focal spot of the photon beam, for quantitative computed tomography with attenuation dynamics of approximately 5 decades, i.e. $1.5 \mathrm{~m}$ of concrete or $40 \mathrm{~cm}$ of steel. Newer detectors (large and continuous line of scintillator needles) are under test to avoid the horizontal sweeping scan of the package imposed by dead layers between the collimated CdTe, and thus to reduce acquisition time. Short-term evolutions include the use of a higher energy and intensity LINAC to interrogate larger and heavier waste packages (up to $5 \mathrm{~T}$ ), and acquisitions at different energies to determine the mean atomic number of objects, by taking advantage of the differences in attenuation according to the photon beam energy spectrum and the atomic number of the elements. In addition to the already measured density, this information will make it possible to refine the identification of the materials.

\subsection{Active neutron interrogation}

Active neutron interrogation is based on the detection of neutrons emitted as a result of fission induced by an external neutron source. The extraction of the useful signal due to induced fission neutrons, embedded in the interrogating flux which is several orders of magnitude higher, uses techniques of time and energy discrimination. This technique can be subdivided into two approaches.

\subsubsection{The method of measuring prompt neutrons}

This method uses a pulse-mode neutron generator to detect prompt fission neutrons induced by thermal neutrons [12]. The principle can be described in three steps:

- the neutron generator emits a short pulse (typically a few dozen to hundred $\mu \mathrm{s}$ ) of neutrons of $14 \mathrm{MeV}$ (during which fissions induced by fast neutrons are produced whose prompt neutrons cannot be used because it is impossible to distinguish them from the interrogating neutrons);

- the fast neutrons of the generator slow down in the constituent materials of the measuring device (notably graphite, see Fig. 15) and the object to be characterised; - when the interrogating flux is essentially thermal (a few hundred $\mu \mathrm{s}$ after the end of the neutron pulse), it becomes possible to discriminate energy between the interrogating neutrons (thermal) and the prompt fission neutrons (fast). This is accomplished with blocks of ${ }^{3} \mathrm{He}$ detectors surrounded by polyethylene, coated with cadmium and/or $\mathrm{B}_{4} \mathrm{C}$ to absorb the interrogating thermal neutrons that will nevertheless let the fast prompt fission neutrons through, the latter being then thermalized by the polyethylene and detected by the ${ }^{3} \mathrm{He}$ counters.

The measured signal contains a component due to prompt fission neutrons proportional to the amount of fissile material. The coefficient of proportionality, called the calibration coefficient, is estimated for the different waste matrices likely to be measured.

\subsubsection{The method of measuring delayed neutrons}

Measurement of delayed neutrons [12] involves two phases, first with an irradiation of the package intended to cause fissions (with fast, epithermal and thermal neutrons) and then a counting of the delayed neutrons resulting from induced fissions. Since each of these phases lasts for several seconds or minutes, the emission of the generator is not necessarily pulsed and the use of an isotopic source of neutrons is possible.

The neutron interrogation method essentially makes it possible to characterise the fissile nuclei $\left({ }^{235} \mathrm{U},{ }^{239} \mathrm{Pu}\right.$ and $\left.{ }^{241} \mathrm{Pu}\right)$. The signal due to fertile nuclei $\left({ }^{238} \mathrm{U}\right.$ and ${ }^{240} \mathrm{Pu}$ ) may nevertheless be significant for some measurements of delayed neutrons (with a harder interrogating flux). Whatever the mode of interrogation (isotopic source, neutron generator), they allow an overall characterisation of the waste package, but they are sensitive to the nature of the matrix (its density and chemical composition), the position of the contaminant in the package and the self-shielding effect in fissile materials. These penalties may be extensive but when these effects are controlled, the method is very sensitive and detection limits can reach a few tens of milligrams of fissile materials. As in the case of passive neutron counting, it is not possible to distinguish the contribution of the different isotopes and the isotopic composition needs to be known a priori (reference spectrum) or determined through other complementary methods (gamma spectroscopy). 

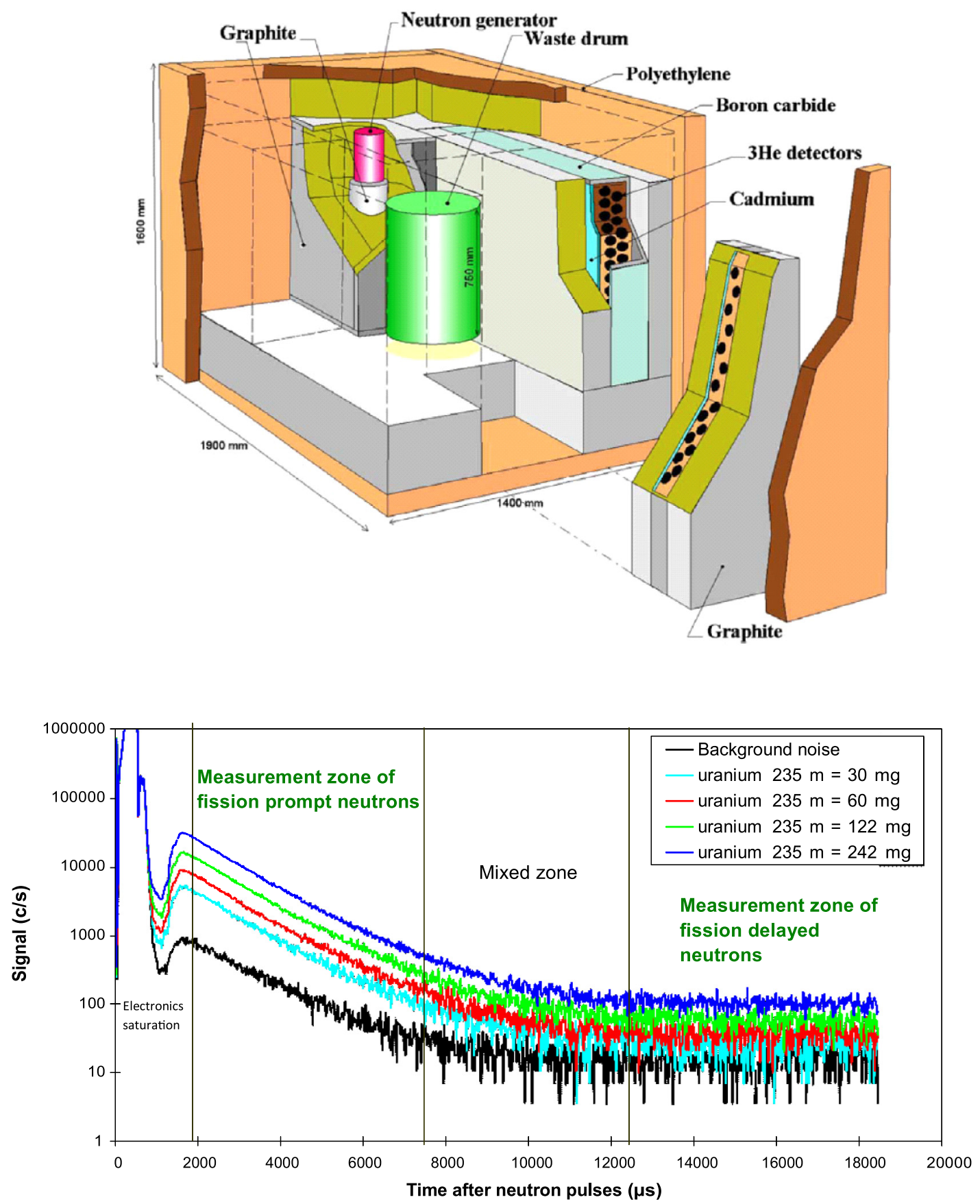

Fig. 15. Above: basic diagram of the PROMETHEE VI neutron interrogation cell at CEA Cadarache; below: time spectrum of the pulsed measurement of prompt and delayed fission neutrons.

Many applications using active neutron interrogation have been developed. For example, the hull compaction facility (ACC) at AREVA La Hague spent fuel reprocessing plant (France) includes two neutron measurement stations. Their main objective is to determine the residual fissile mass in the hulls after dissolution of the fuel (before compacting) and in the final container of compacted waste (see Fig. 16). Coupled to gamma spectrometry, active neutron interrogation allows advanced characterisation of these waste drums. Note that in passive mode, i.e. when the neutron generators are off, the neutron measurement stations also allow assessing the curium activity, by detecting its dominant spontaneous fission neutron emission.

\subsection{Active photon interrogation (Photofission)}

The use of high-energy photons and of the photofission phenomenon, a physical phenomenon similar to neutron fission but induced by photons with energy greater than $6 \mathrm{MeV}$, allows the actinides to be assayed within a waste package. In the same way as high-energy photon imaging 

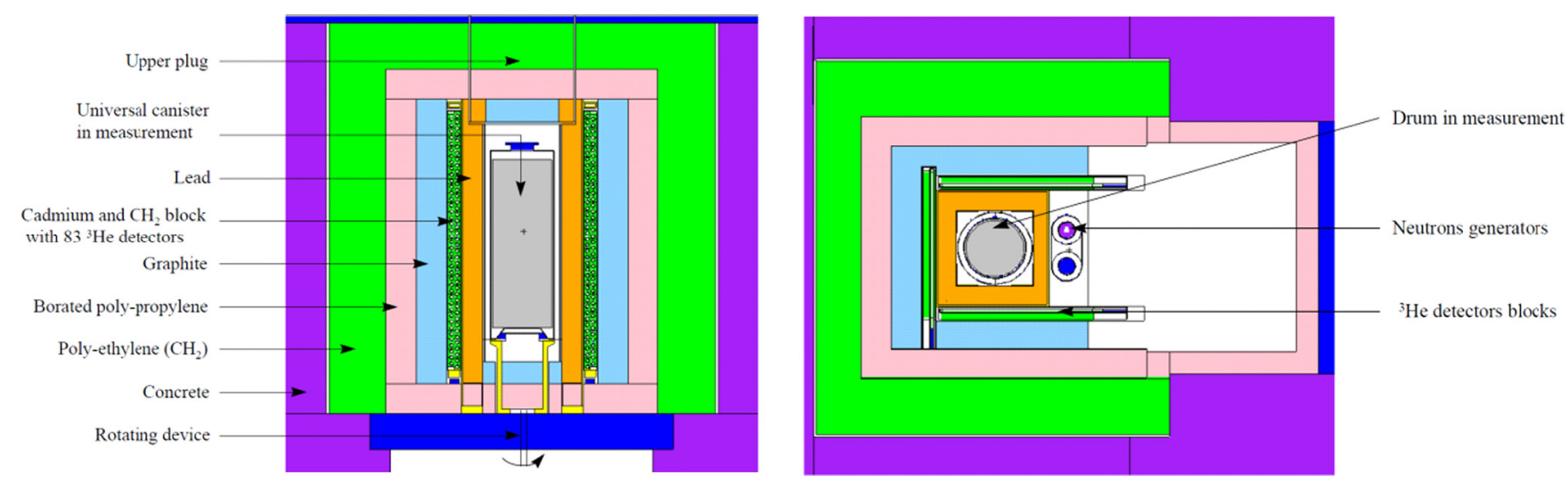

Fig. 16. Numerical model of the ACC neutron interrogation station dedicated to the CSD-C compacted waste drums of the ACC compaction facility at AREVA La Hague [13].

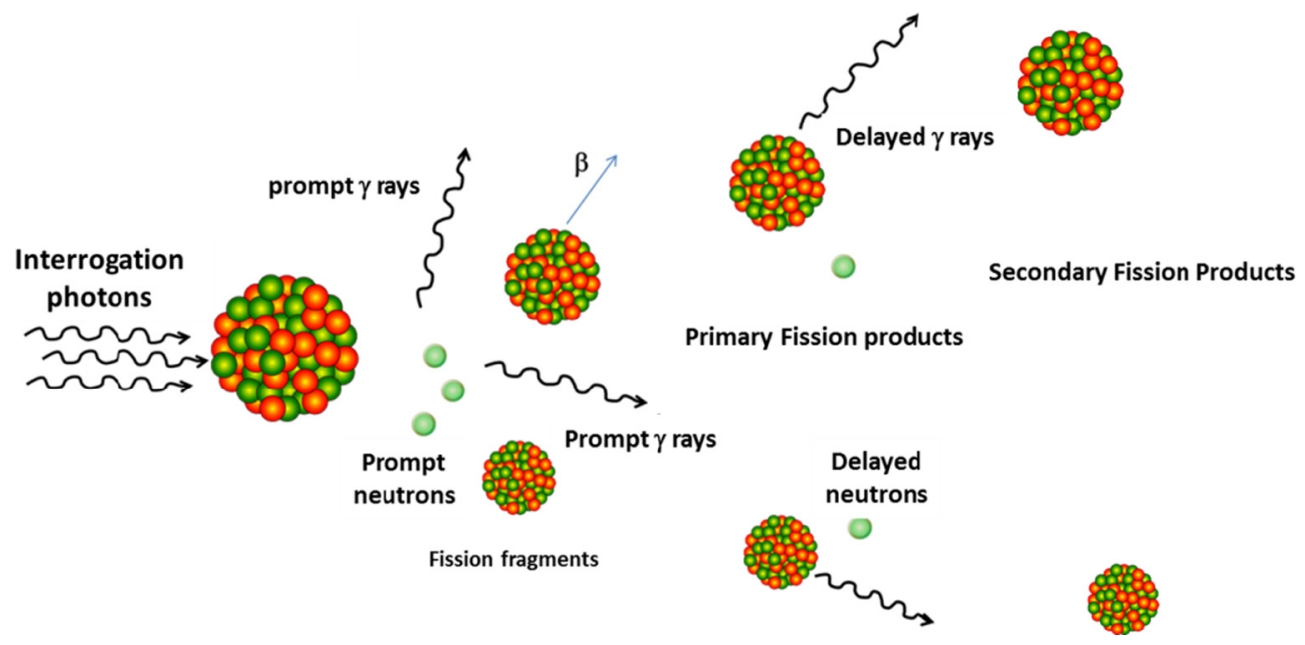

Fig. 17. Basic diagram of photofission.

described above, this method uses a high-energy photon beam produced with a LINAC and a conversion target (Bremsstrahlung). The photons, beyond threshold energy of about $6 \mathrm{MeV}$, have the power to cause the fission of heavy nuclei with a mass greater than that of lead (see Fig. 17).

By knowing the characteristics of the interrogating high-energy photon beam (energy, intensity, direction...), the measurement of delayed neutrons or gamma radiations from photofission allows the dosage of the quantity of actinides present in a radioactive waste package, photofission prompt particles being difficult (prompt neutrons) or impossible (prompt gamma rays) to detect due to the intense background following the LINAC pulses.

The photofission cross-section exhibits a maximum for photons of $15 \mathrm{MeV}$ energy (exciting the giant dipole resonance of the target nucleus, with decreasing of the said resonance by the fission path). In contrast to thermal neutron fission, all actinides are likely to undergo photofission with similar probability, even isotopes ${ }^{234} \mathrm{U},{ }^{238} \mathrm{U}$, $\left.{ }^{238} \mathrm{Pu},{ }^{240} \mathrm{Pu},{ }^{242} \mathrm{Pu}\right)$ as well as the odd ones $\left({ }^{233} \mathrm{U},{ }^{235} \mathrm{U}\right.$, ${ }^{239} \mathrm{Pu},{ }^{241} \mathrm{Pu}$ ) of uranium and plutonium.
The first photofission waste characterisation studies developed at the CEA exploited the detection of delayed neutrons. The methods used to detect delayed gamma rays are more recent [14]. Evaluations on waste packages were carried out in particular at the SAPHIR facility in CEA Saclay (Saclay photonic activation and irradiation activation system, Fig. 18) and continued at CEA Cadarache, by simulation and experimentation in the CINPHONIE facility (photonic and neutron interrogation cell, Fig. 13, Sect. 3.2 on high-energy photon imaging) with an electron LINAC (currently $9 \mathrm{MeV}$, short term, $20 \mathrm{MeV}$ ).

Delayed neutrons are emitted by the precursor nuclei of fission products up to a few tens of seconds after photofission. They are counted during irradiation, between each pulse of the LINAC.

The prompt neutrons of photofission are produced a few fractions of picoseconds after photofission, with a production rate much higher than that of delayed neutrons (about a factor of 100) but their detection is made very difficult by the intense photon flash of the LINAC, accompanied by an intense photo-neutron flux that blinds the generally used ${ }^{3} \mathrm{He}$ neutron counters. New types of activation sensors, 

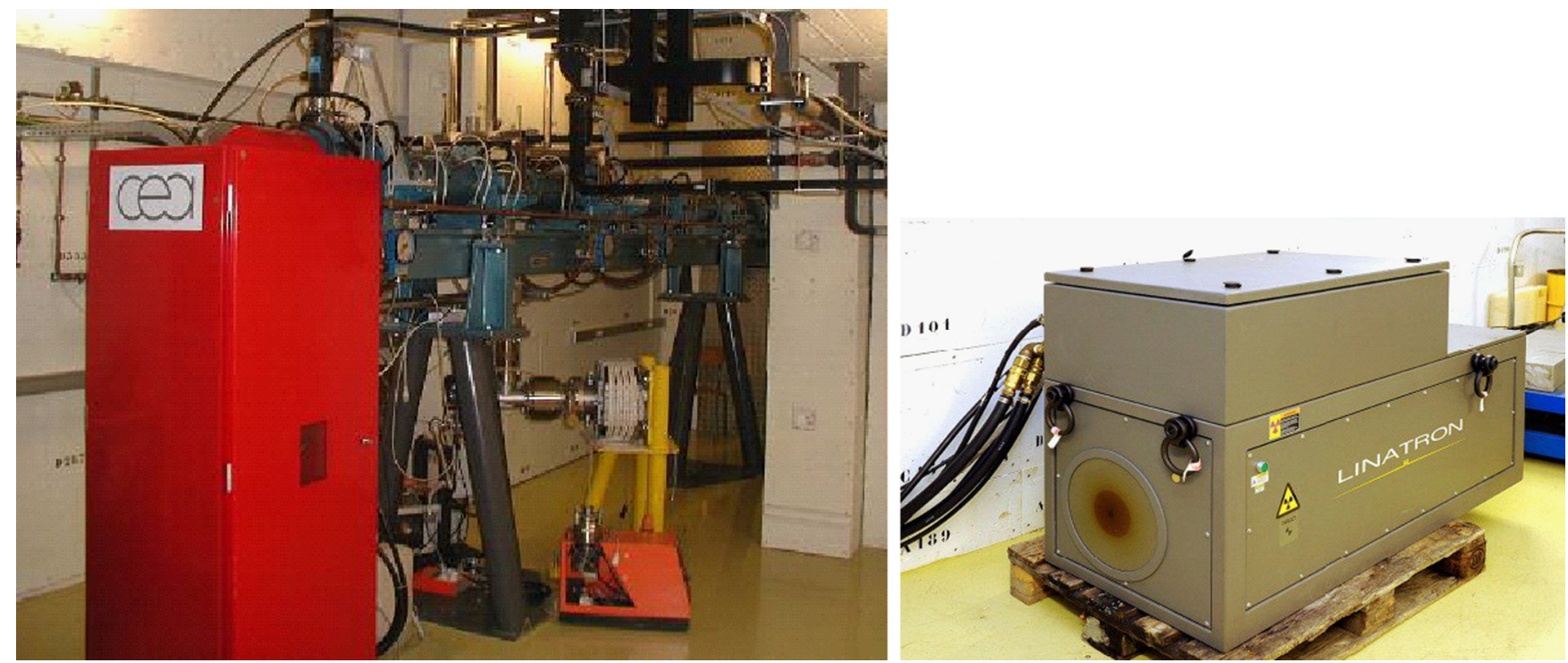

Fig. 18. The SAPHIR facility of CEA LIST makes it possible to characterise waste packages by photofission (A. Gonin/CEA). Left: $15 \mathrm{MeV}$ LINAC. Right: 6/9 MeV LINAC.

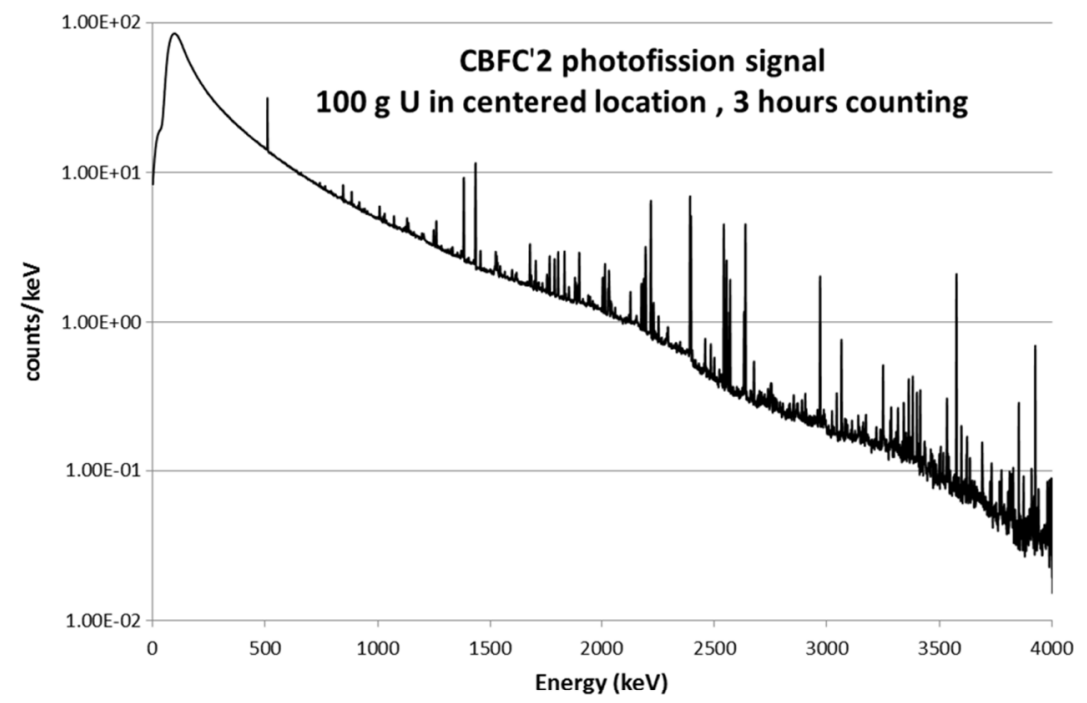

Fig. 19. Simulated spectrum of delayed gamma rays of photofission for a $100 \mathrm{~g}$ uranium sample in centred position in a CBFC'2 waste package.

including fluorine for instance, are now being studied to gain access to information from prompt neutrons, not just after the LINAC pulses but after stopping irradiation, in view to provide additional data in the interpretation of photofission measurements.

As mentioned above, the measurement of delayed gamma rays of fission is the only possible one, the prompt gamma rays being masked by the intense photon flash of the LINAC pulse. Today it is applied after irradiation in two forms: on the one hand in total high energy gamma counting $\left(E_{\gamma}>3 \mathrm{MeV}\right)$ using detectors with high stopping power (BGO scintillator), and on the other hand in HPGe. The method has been evaluated in particular by tests on package mock-ups and real packages by global gamma counting. On the other hand, Figure 19 shows the spectrum of the delayed gamma rays of a $100 \mathrm{~g}$ uranium sample centred in a large concrete package (about $1.2 \mathrm{~m}^{3}$ ), obtained by a Monte Carlo simulation for a $2 \mathrm{~h}$ irradiation with a $15 \mathrm{MeV}$ LINAC followed by a 3 hour post-irradiation counting period using a HPGe detector.

The use of multiple detectors, placed all around the examined package, also allows tomographic information to be produced from high-energy delayed neutrons or gamma rays [14]. This photofission tomography technique offers the possibility to locate nuclear material that has undergone photofission within the waste package, after computer reconstruction of the detectors data, and to thereby reduce the uncertainties by making it possible to focus the interrogation and the detection on the specific zone of interest in case of hot spot. 


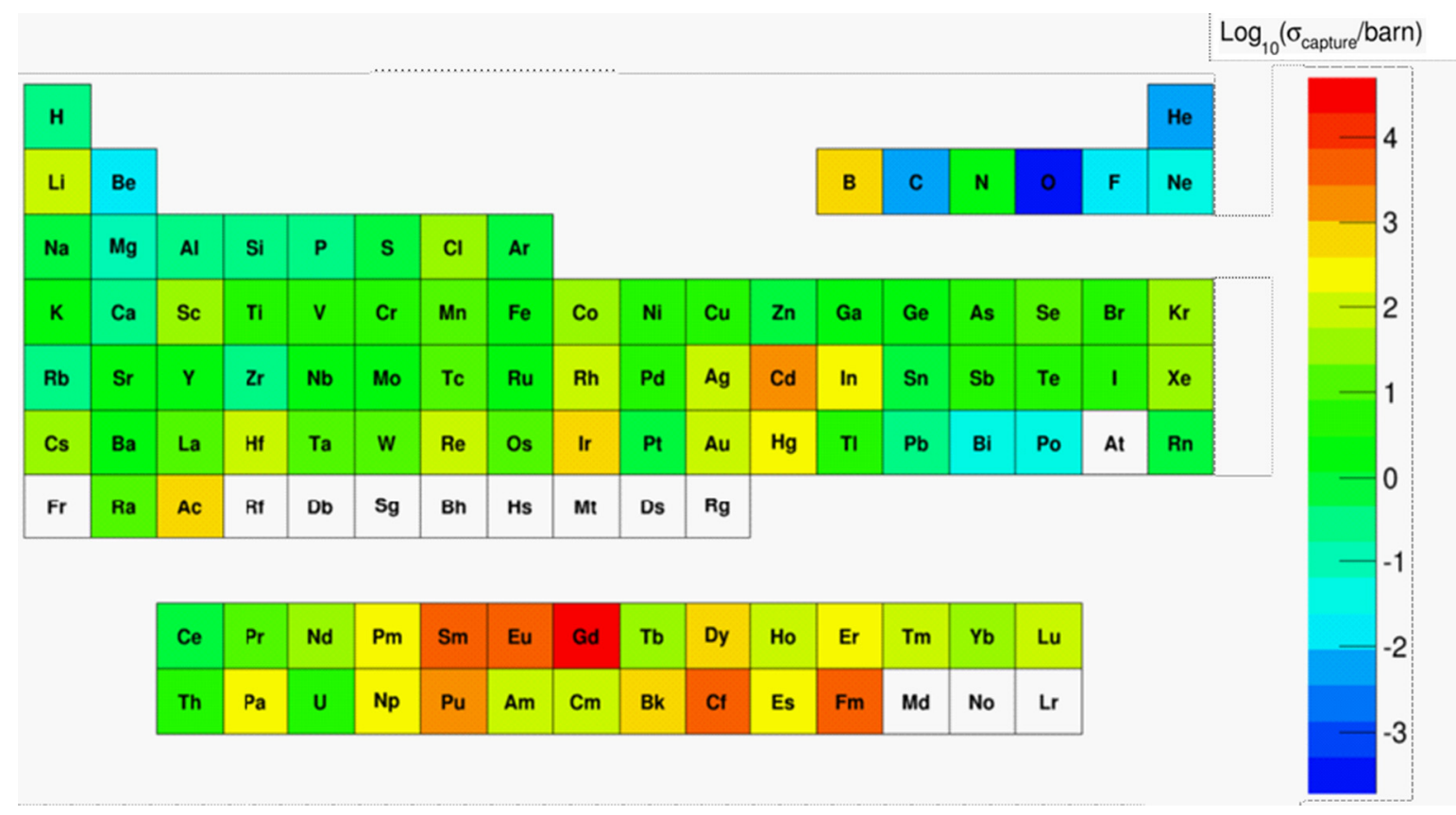

Fig. 20. Cross sections of radiative capture for thermal neutrons.

As mentioned above, the fissile actinides $\left({ }^{235} \mathrm{U}\right.$ and $\left.{ }^{239} \mathrm{Pu}\right)$ and fertile ones $\left({ }^{238} \mathrm{U}\right)$ undergo photofission with a probability of the same order of magnitude. Their discrimination is therefore necessary to estimate certain quantities of interest such as the fissile matter mass. Different methods are possible, such as the analysis of delayed gamma ratios $[15,16]$, the time evolution of the delayed gamma signal, or the ratio of delayed gamma and neutron signals.

Photofission is a promising technique for the characterisation of large-volume concrete waste packages for which other non-destructive nuclear measurement methods are reaching their limits. The feasibility studies referred to above are therefore being pursued as part of a major development programme to bring it to the industrial application stage.

\subsection{Prompt gamma neutron activation analysis}

Many nuclei are identifiable by their prompt gamma radiation induced by neutron activation (PGNAA) in particular that which is emitted after the radiative capture $(n, \gamma)$ of a neutron, the reaction being all the more likely as the energy of the neutron is low. It is generally characterised by the cross section of radiative capture with thermal neutrons $(0.025 \mathrm{eV})$, of which Figure 20 gives an order of magnitude for most elements of the Mendeleev periodic classification, taking into account their natural isotopes. If a few elements such as boron, gadolinium, cadmium or mercury have a very high radiative capture cross-section of more than 100 barns, most elements such as iron, chlorine or nickel have a lower cross-section, of the order of the barn, which nevertheless authorises their characterisation by measuring their prompt gamma radiation. Only a few elements such as carbon, oxygen or lead have capture cross-sections that are too low to be assayed by PGNAA. For these elements, reactions involving fast neutrons can be preferred, such as inelastic scattering (n, n' $\gamma$ ) that also produces gamma rays characteristics of the interrogated elements. Detection limits are however much smaller because cross sections are well below 1 barn for all the elements, and the larger fast neutron flux (compared to the thermal flux) does not compensate for that.

Since interrogation sources produce fast neutrons of a few MeV, they are surrounded, as well as the analysed object, by a moderating material to promote capture reactions at thermal energy. The most commonly used neutron generators are based on the DD or DT fusion reaction, which produces $2.5 \mathrm{MeV}$ or $14 \mathrm{MeV}$ neutrons, respectively, depending on whether the target on which the deuterium ions are accelerated contains deuterium or tritium. These generators operate in pulsed mode, which makes it possible to favour the measurement of the radiative capture gamma radiations between the pulses, once the neutrons have been thermalized to eliminate the noise of the abovementioned reactions with fast neutrons, such as inelastic scattering $\left(n, n_{\gamma}{ }^{\prime}\right)$. Indeed, the later may induce a significant background on the major elements constituting the waste or the measurement system, when capture gamma rays of minor elements (traces) are searched. Gamma radiation is analysed by high resolution spectrometry with an HPGe detector. An example of a measuring cell operating on this principle is shown in Figure 21 [17] and examples of gamma spectra are reported in Figure 22 [18].

PGNAA can be used to characterise radioactive waste, revealing the presence of toxic or reactive substances (boron, chlorine, cadmium, gadolinium mercury, aluminium, nuclear materials, etc.), or the presence of neutron moderators (carbon, hydrogen) and neutron absorbers (boron, cadmium, gadolinium) in order to correct neutron 


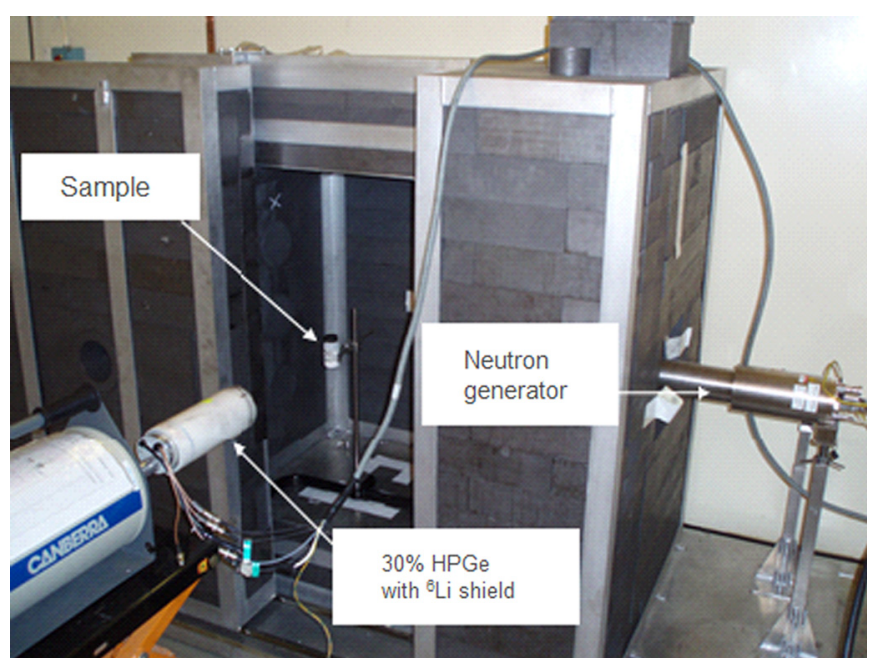

Fig. 21. The REGAIN system for PGNAA measurements at CEA Cadarache, using graphite to moderate the $14 \mathrm{MeV}$ neutrons delivered by a pulsed DT neutron generator with a $10^{8} \mathrm{~s}^{-1}$ neutron emission, and a HPGe detector to assay the neutron-induced gamma rays [17].
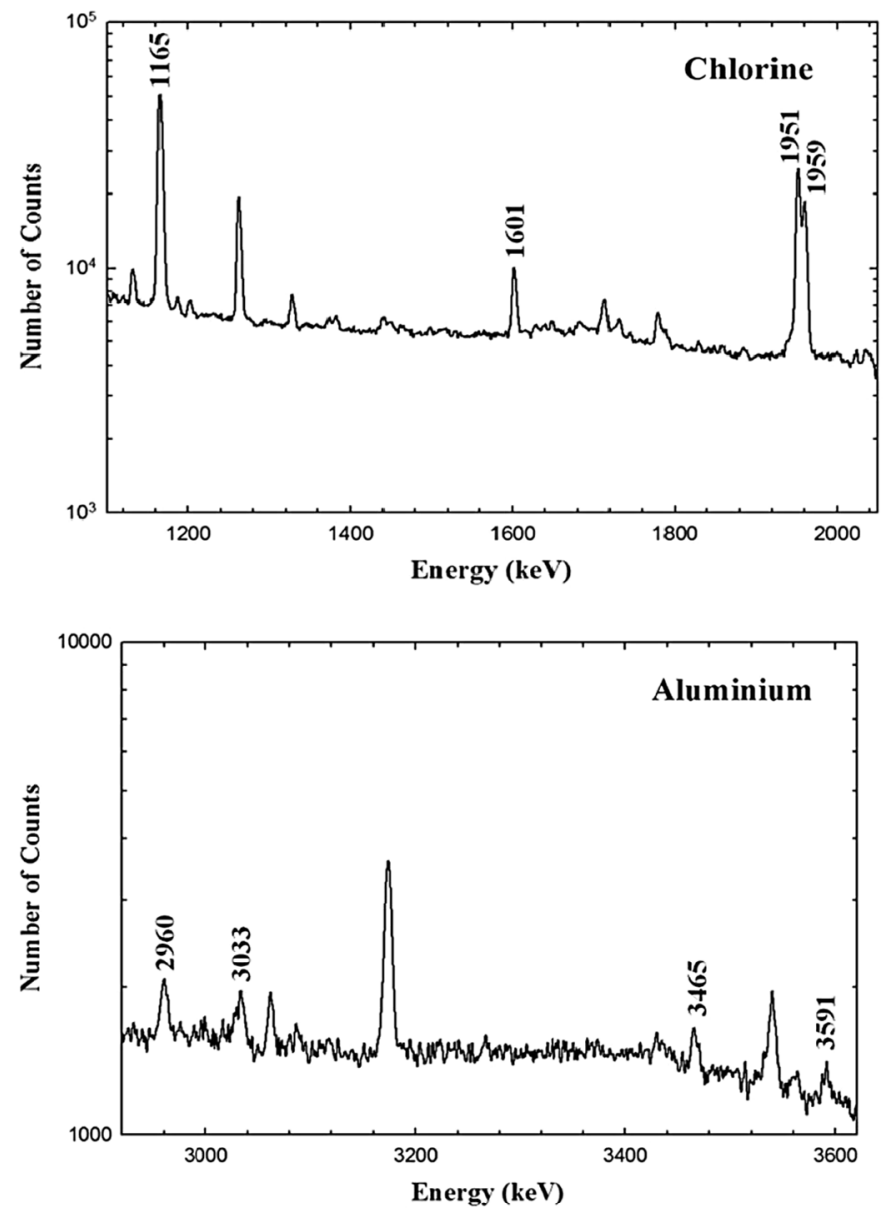

measurements for matrix effects in a waste package. This technique is also applied for online analysis of cement- or coal-based crusts, in petroleum prospecting, and its use is also being considered to retrieve precious metals and rare earths from electronic waste (computers, mobile phones, etc.). It is even possible to characterize nuclear materials through neutron activation by measuring delayed gamma radiation of the induced fissions, between the pulses of the generator or after irradiation according to the radioactive period of the fission fragments emitting these radiations [19].

\section{Destructive measurements for radioactive waste package characterisation}

The destructive measurement is an indispensable complement to the non-destructive measurement of radioactive waste, particularly for historic waste packages with little or insufficient available data. It is also used for recent packages or different types of recent waste as part of quality controls such as "Super controls" (SCO), see Section 5.
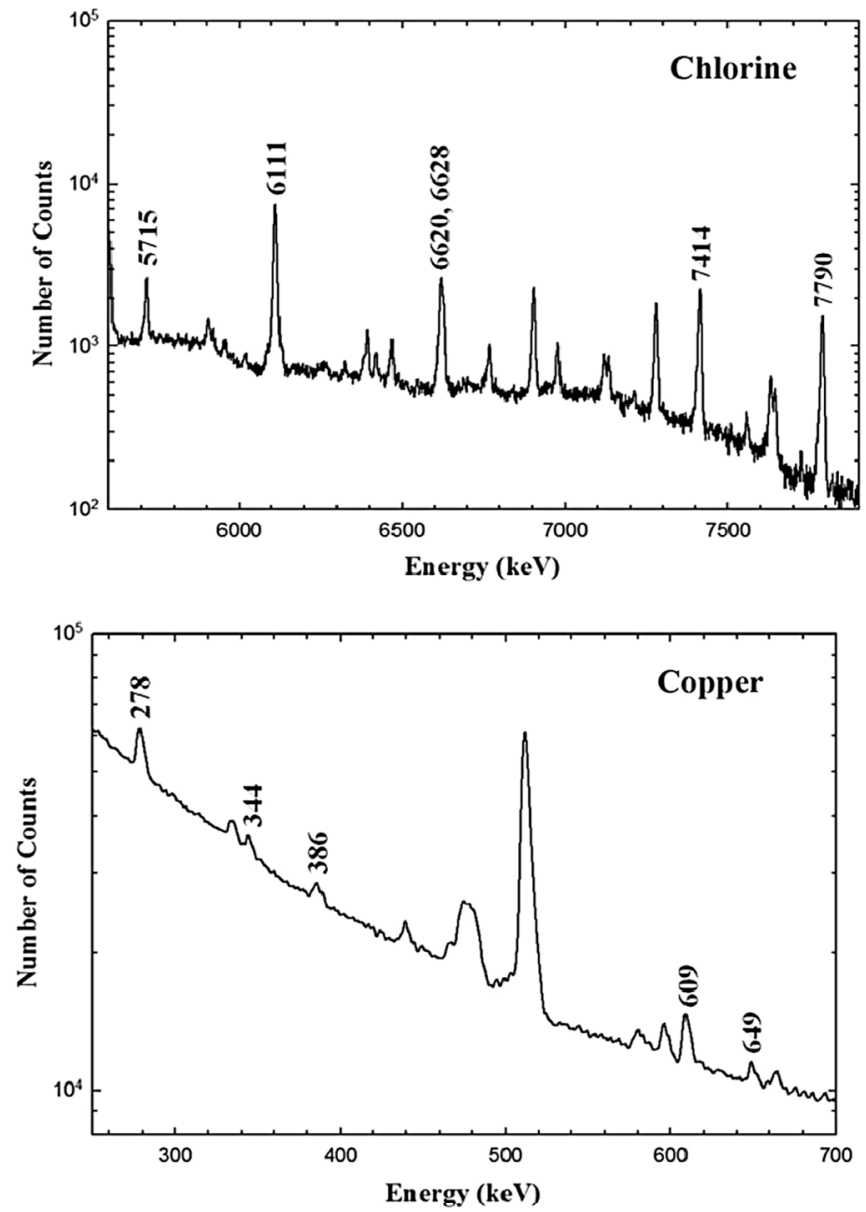

Fig. 22. Examples of neutron activation spectra showing the main gamma radiative capture lines in chlorine, aluminium and copper samples [18]. 
Destructive measurement can be broken down into three phases: (1) expertise, (2) sampling and sample preparation, and (3) measurement on samples.

\subsection{Destructive appraisal and sampling}

The form of destructive expertise will depend on the type of waste packages to be appraised. Packages can be classified into two types: (1) homogeneous waste packages (e.g. ion exchange resin embedded in resin, or concentrates from an effluent treatment plant coated in a hydraulic binder or bitumen), and (2) heterogeneous waste packages (e.g. bulk waste immobilized by mortar, or drums reconditioned in a box-shaped container). For producers, as part of quality control procedure, there may be a third type of nonimmobilized waste package (e.g. Intermediate and LowLevel Short-Lived radioactive waste, which is injected by ANDRA at the Aube Centre, a surface repository located in France).

For homogeneous packages, the expertise technique is coring. In order to carry out chemical and radiological analyses on the samples taken, the coring must be carried out in air and not by cooling with water in order to avoid leaching the materials, especially the radioactive ones. This technique makes it possible to carry out the expertise, namely the observation of the package (i.e. the presence of the various components of the package, the quality of the embedding and the measurement of the apical vacuum). At the same time, samples can be taken.

For heterogeneous packages, the reference expertise technique is cutting. Indeed, it enables us to observe the interface between the various components of the package and in particular between the blocking binder and the waste. On the other hand, this technique does not allow sampling (other than punctually on incorrectly blocked waste). It is therefore logically associated with coring means, especially for the concrete casing or the blocking mortar. Regarding the analysis of the waste, it is possible to make smears (or even retrieve some pieces on some types of waste) or to implement non-destructive techniques such as gamma spectrometry or the alpha camera with good results, since there is no longer a barrier between the detector and the waste.

For unblocked waste, the appraisal consists of an exhaustive inventory of the waste contained in the package, the sorting of the objects according to their physical nature and/or their level of activity (irradiation and/or contamination).

The actual expertise provides a significant amount of information on package structure and manufacturing quality. The visual observation of the cores or of the cut face in the case of heterogeneous waste, reveals the presence of the various constituents of the package (conventionally a concrete envelope, biological protection, the immobilized waste in a matrix), the quality of its various constituents (homogeneity of the waste or concrete of the envelope or that of the blockage) as well as the interfaces between the latter elements, the presence of vacuum (absence of blocking mortar in certain places or bad filling), or even the presence of free water or prohibited waste.
The CEA has two shielded cells (ALCESTE, see further in Fig. 30, and CADECOL here in Fig. 23) dedicated to destructive expertise on a wide variety of waste packages at the CHICADE facility in Cadarache. These heavy means, which are unique in France, are necessary because once the integrity of the package has been destroyed and the waste has been exposed, the risks of contamination and/or irradiation require working in remote control with nuclear ventilation, biological protection and adapted handling facilities.

Waste disposal after appraisal is a problem in its own right. Cored packages are no longer in compliance with the specifications for handling by storage or storage facilities. It is therefore necessary to recondition them in new packages acceptable for storage. For cut packages, the pieces must be reconditioned in packages compatible with the nature of the waste. In order to accomplish this, the number and location of cuts may be dictated by reconditioning.

\subsection{Analyses on samples}

The destructive expertise makes it possible to take samples of the various components in the package. It is then possible to carry out analyses or measurements on these samples.

The representativeness of the samples is a key parameter since the cost of the measurements does not allow the analyses to be multiplied. As for the measurements of physical characteristics, it is important to select samples that show no singularity. On the other hand, for the radiological analyses, the most active samples are generally selected. The result obtained can thus be considered as envelope and therefore higher if its purpose is to estimate the average activity of the package.

\subsubsection{Samples of the package envelope}

Conventionally, measurements on the materials of the external envelope of the heterogeneous waste package or binder are devoted to the mechanical and containment properties of the material.

\subsubsection{Mechanical properties}

Specimens, in accordance with the standards in force, are made by cutting in the cores to conduct tests of resistance to compression.

\subsubsection{Containment properties}

With regard to the containment, the material must prevent the radioelements in the solid waste from passing through the package envelope and migrating outwards. Since the vector of this transport is water, its velocity in the material is therefore considered to be greater than that of the radioelements. The desired containment property is therefore the diffusion coefficient of the tritiated water, tritium being used as a tracer for the measurement [20]. Since the materials are either inactive or slightly contaminated, the tests can be carried out under a hood or in a glove box. The principle of the method (illustrated in 


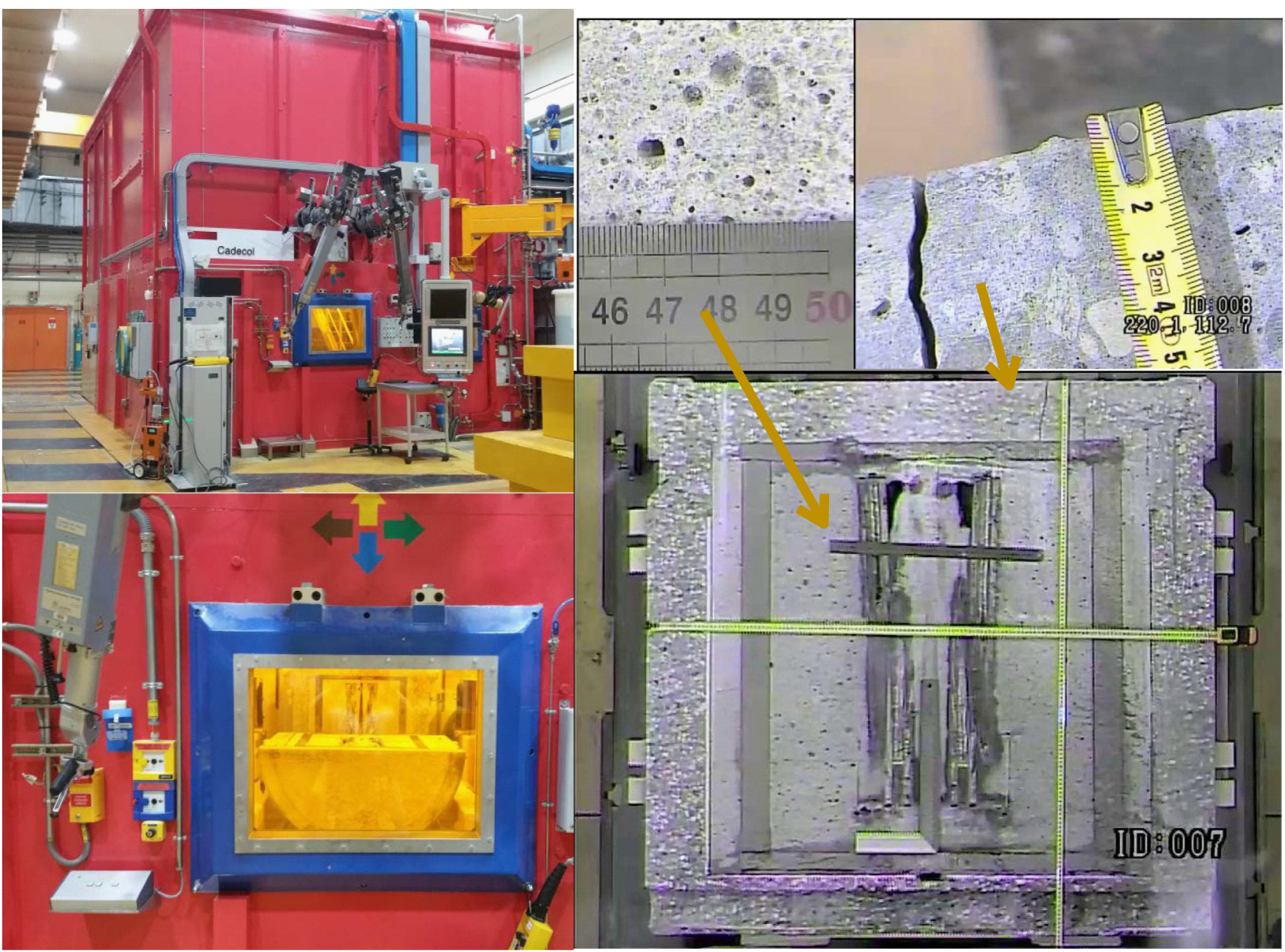

Fig. 23. Expertise of a heterogeneous hull (cutting of a $5 \mathrm{~m}^{3}$ concrete package by wet process) in the CADECOL cutting and coring hot cell, in CHICADE basic nuclear facility, at CEA Cadarache.

Fig. 24) is to measure the diffusion kinetics of the tritiated water through a test specimen of the material placed between two compartments, the first with tritium and the second in which the activity of the tritium having passed through the test specimen is measured.

The measurement of gas permeability also makes it possible to account for the capacity of the material to confine the radioactive gases. For this purpose, a test specimen of the material is placed in a reactor guaranteeing leak tightness on the side of the test specimen. The application of a nitrogen pressure on one side of the test specimen and the measurement of the gas flow rate on the opposite side makes it possible to calculate a permeation coefficient depending on the relative humidity of the material (see Fig. 25). Beyond these sample tests, it is also advisable to measure the gases emitted by the package directly or by the waste block as described in Figure 3 in Section 2.5.

\subsubsection{Waste samples}

For the homogeneous waste material, it is necessary to check the mechanical strength and containment characteristics, as well as the radiological information of the waste as well as the chemical analysis of certain compounds such as toxic chemicals or organic complexing agents which may accelerate the transport of certain radioelements.

\subsubsection{Containment properties}

The purpose of containment is to prevent the release of radionuclides from the matrix. In this case, a leaching test is used. A sample of the material is placed in a solution which is periodically analysed to determine the transfer rate of radioelements from the waste matrix to the solution. For gaseous radioelements, gas permeability tests are also carried out on the homogeneous waste matrix.

Mechanical strength tests or containment property measurements involve significant quantities of waste. The quantity of $\alpha$ emitters or the level of irradiation means that these tests are necessarily performed in a glove box or in a shielded cell.

\subsubsection{Radiochemical and chemical measurements}

Radiochemical and chemical measurements ensure compliance with specifications as well as the conformity of waste packages. The implementation of this characterisation 


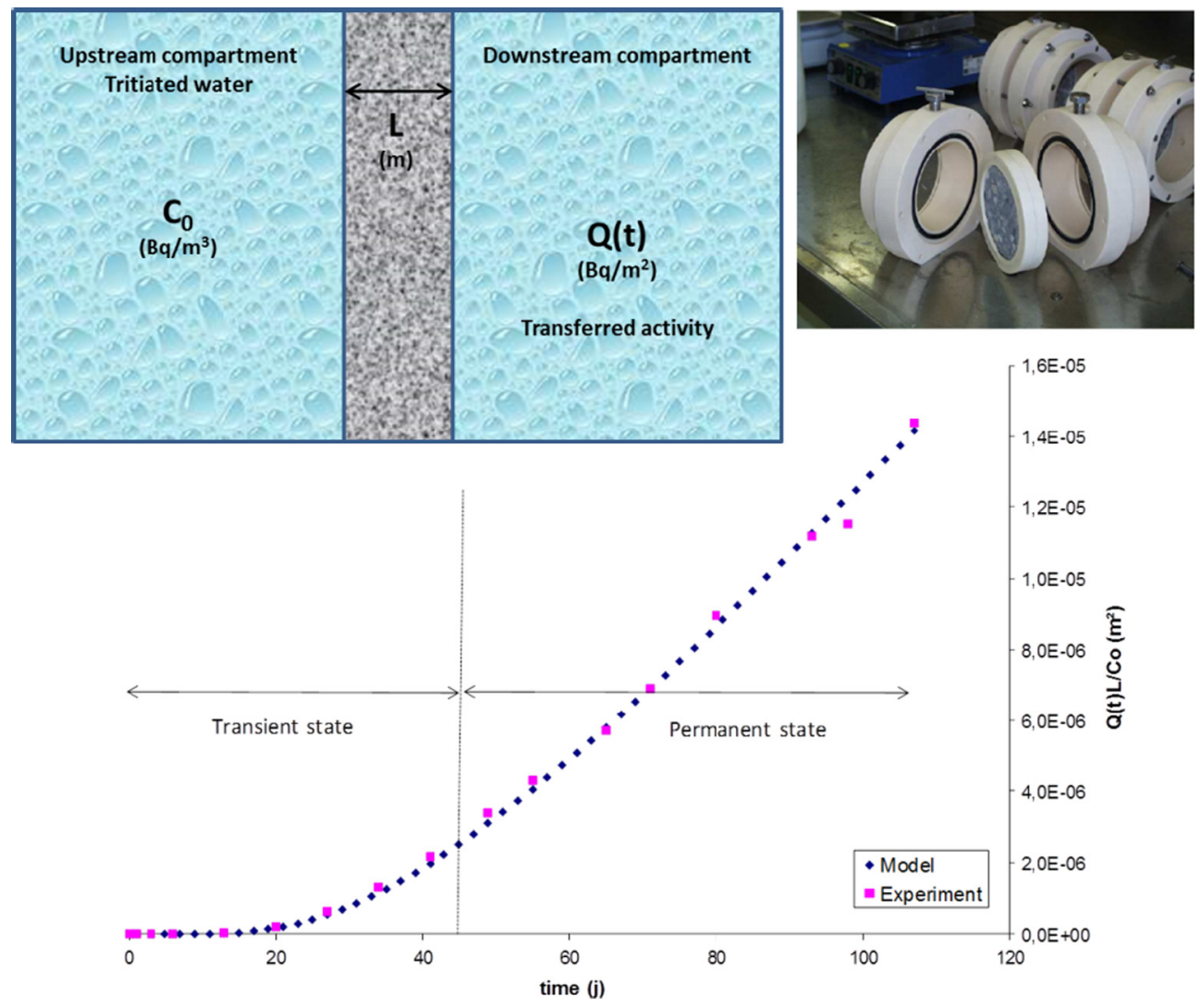

Fig. 24. Measurement of the effective $D_{e}$ diffusion coefficient of the concrete using the Fick law.

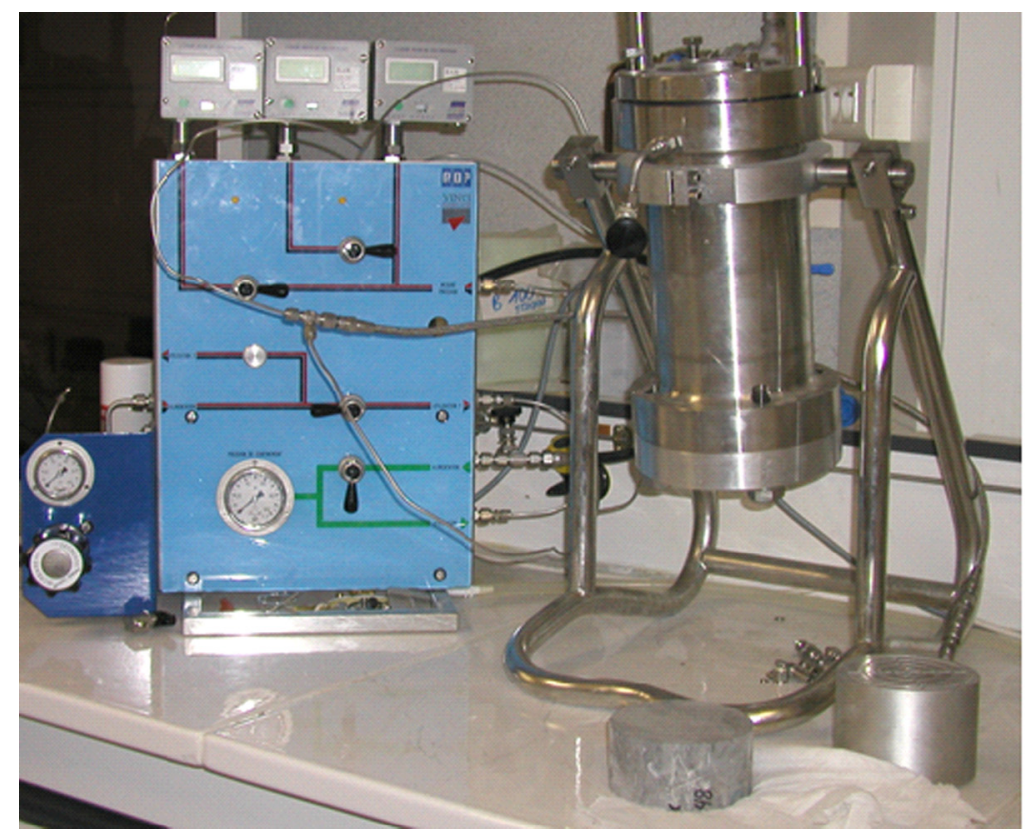

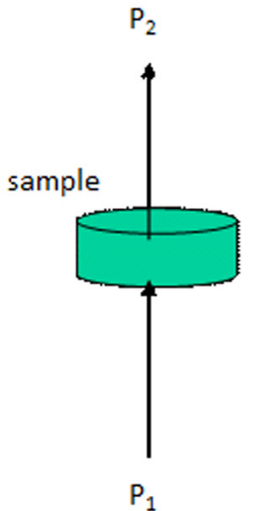

nitrogen

$$
k=\frac{2 Q L P \mu}{S\left(P_{1}^{2}-P_{2}^{2}\right)}
$$

$L$ thickness of sample $(\mathrm{m})$ $\mu$ dynamic viscosity of gas (Pa.s) $\mathrm{P}_{1}$ applied inlet pressure $(\mathrm{Pa})$ $\mathrm{P}_{2}$ outlet pressure $(\mathrm{Pa})$ $\mathrm{P}_{0}$ atmospheric pressure $(\mathrm{Pa})$ $S$ surface area $\left(\mathrm{m}^{2}\right)$ Q measured flow rate $\left(\mathrm{m}^{3} / \mathrm{s}\right)$

Fig. 25. Measurement of gas permeability. The concrete test specimen is placed in the cell and leak tightness on the sides of the test specimen is ensured by a pressure of 60 bars. Afterwards, a pressure of 2 bars is applied to the bottom side of the test specimen and the flowrate crossing through the specimen is measured. 


\section{Analytical process}

\section{SAMPLES}

SAMPLE PREPARATION

DEVICES for grinding, cutting, homogenization of samples (from $\mathrm{kg}$ to $\mathrm{g}$ ) adapted to the matrix and activity
Homogeneity control : gamma spectroscopy on solid samples
Sample destruction

Mineralization (acid, alkaline fusion) or heat treatment (combustion)

Requires generally a sample in the form of powder (a few grams)

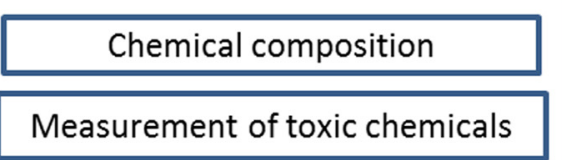

Gamma spectroscopy $\left({ }^{60} \mathrm{Co}\right.$, ${ }^{137}$ Cs..) and alpha spectroscopy

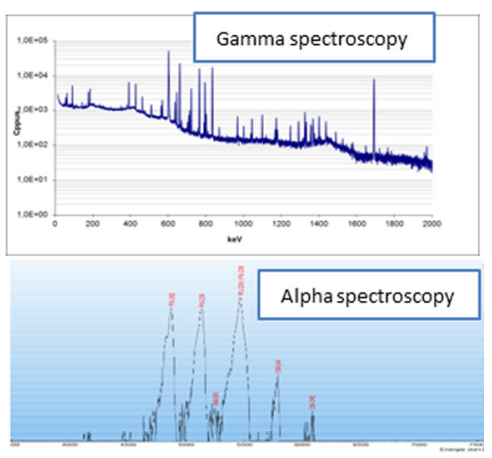

SELECTIVE SEPARATION

Method: precipitation, liquid-liquid extraction, Ion Exchange Chromatography ...

Necessity to use several methods to obtain sufficient decontamination and to ensure a selective

measurement of the radionuclide. These methods must be adapted to the dissolution media and the

measurement technique

\section{Measurements}

\section{Isotopic techniques}

ICP/MS or ICP/MCMS

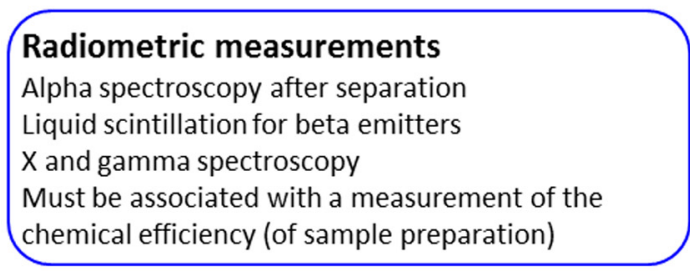

\section{Measurements of toxic chemicals}

ICP/AES, ICP/MS

\begin{tabular}{|c|c|c|}
\hline Radionuclide & $\begin{array}{c}\text { Radioactive } \\
\text { half-live (years) }\end{array}$ & $\begin{array}{c}\text { Measurement } \\
\text { Technique }\end{array}$ \\
\hline${ }^{10} \mathrm{Be}$ & $1,4.10^{6}$ & ICP/MS \\
\hline${ }^{14} \mathrm{C}$ & $5,7,10^{3}$ & S.L. \\
\hline${ }^{36} \mathrm{Cl}$ & $3.0 .10^{5}$ & S.L. \\
\hline${ }^{41} \mathrm{Ca}$ & $1.10^{5}$ & S.L. \\
\hline${ }^{59} \mathrm{Ni}$ & $7,6.10^{4}$ & $\mathrm{X}$ \\
\hline${ }^{63} \mathrm{Ni}$ & 100 & S.L. \\
\hline${ }^{79} \mathrm{Se}$ & $3,6.10^{5}$ & LC-ICP/MS \\
\hline${ }^{93} \mathrm{Mo}$ & $4.10^{3}$ & $\mathrm{X}$ \\
\hline${ }^{94} \mathrm{Nb}$ & $2,0.10^{4}$ & Gamma \\
\hline${ }^{99} \mathrm{TC}$ & $2,1.10^{5}$ & LC-ICP/MS \\
\hline${ }^{107} \mathrm{Pd}$ & $6.10^{6}$ & LC-ICP/MS \\
\hline${ }^{108 \mathrm{~m}} \mathrm{Ag}$ & 440 & Gamma \\
\hline${ }^{129} \mathrm{I}$ & $1,6.10^{7}$ & MC-ICP/MS \\
\hline${ }^{121} \mathrm{Sn}$ & 55 & Gamma/X \\
\hline${ }^{126} \mathrm{Sn}$ & $2.10^{5}$ & ICP/MS \\
\hline${ }^{135} \mathrm{Cs}$ & $2.3 .10^{6}$ & LC-ICP/MS \\
\hline${ }^{151} \mathrm{Sm}$ & 90 & S.L. \\
\hline
\end{tabular}

Fig. 26. Radiochemical and chemical analysis strategy of samples taken from a waste package.

programme requires an analytical strategy illustrated in Figure 26, combining different measurement techniques with chemical separation and concentration methods.

This strategy takes into account the nature of the matrix, that of the primary waste (homogeneous or heterogeneous) and its conditioning, its level of activity, the isotopes to be sought (radiological spectrum, contamination, radionuclides or volatile elements). The analytical program is generally divided into three parts which are the following:

- Measurements of the main radionuclides at the time of characterisation; 
- Measurement of long-lived radionuclides, activities that are weaker but crucial for long-term storage;

- Measurement of toxic chemicals.

For solid waste, after optional grinding and homogenization, sampling solubilizing is necessary, the analyses being generally carried out on aqueous solutions. It must be adapted to the matrix (waste and embedding medium) and depends on the radionuclides or the element to be dosed. It is for this reason that complementary techniques can be used, such as dissolution by various acid media with retrieval of the volatile elements and a heat treatment (or even combustion). These multiple mineralisation processes ensure the total solubilisation of all elements or radionuclides, and more especially the volatiles ones. Furthermore, the selected sample to be analysed must be representative of the whole waste sample.

Few analyses are possible on mineralization solutions without preparation. These are mainly gamma spectrometric and toxic chemical measurements.

Extraction protocols using precipitation, liquid-liquid extraction or ion exchange chromatography methods are then applied depending on the radionuclides (chemistry of the element) and on the measurement technique, whether it is radiometric or isotopic. They make it possible to separate the interfering radioisotopes and to concentrate the radionuclide to be measured. These extraction methods are specific to each emitter and matrix and can be combined to obtain perfectly decontaminated solutions, depending on the complexity of the mixture and the instrumental technique.

The resulting solutions are then subjected to isotopic characterisation measurements. These techniques are based either on the disintegration properties (measurements of the beta emitters by ultra-low liquid background scintillation, gamma or alpha emitters with high-performance spectrometry chains, etc.), or on their specific mass (measurement by inductively coupled plasma - mass spectrometry, ICP-MS).

In addition to the gamma and alpha emitting isotopes, the most sought-after pure beta emitters are tritium, carbon 14, nickel 63 or strontium 90. They are measured in liquid scintillation.

By analysing various samples, it becomes possible to establish the characterisation of the waste package and to estimate its homogeneity. The same analytical scheme can also be applied to the waste before its processing.

\section{The combination of measurement methods}

Non-destructive, passive and active methods of measurement and destructive methods have complementarities used in the characterization of radioactive waste packages. Within this framework, SCO are second-level examinations carried out at the request of ANDRA on certain low- and medium-activity, short-lived (FMA-VC) waste packages destined for surface Storage at the Aube Centre (CSA). They enable operators to check the conformity of the geometrical, radiological, physical and chemical character- istics of the packages in accordance with the acceptance criteria of the CSA thanks to:

- Non-destructive measurement techniques: radiography/ tomography, gamma spectrometry, passive/active neutron measurements, and ${ }^{3} \mathrm{H}$ and ${ }^{14} \mathrm{C}$ degassing measurements;

- destructive measurement techniques: coring and cutting in order to obtain samples and afterwards conduct chemical analyses of toxic and radiochemical radionuclides of interest and physical tests for porosity measurement, permeability, diffusion coefficients, mechanical strength and leaching.

The objective is to verify that the geometrical, radiological, physical and chemical characteristics of the packages comply with the mandatory specifications and the descriptions of the CSA accreditation files. SCO require combining the different measurement techniques for the most complete characterisation of packages and also to reduce measurement uncertainties. Non-destructive testing combines physical characterization techniques using high-energy photon imaging (radiographies and tomographies) and gamma spectrometry along with passive and active neutron measurements. The choice of techniques and their coupling depends on the characteristics of the package (its mass, volume, matrix, reported radionuclides and their $\alpha, \beta$ activities, etc.), expectations of the expertise ( $\alpha$ activity after 300 years, total $\alpha, \beta$ activities) with a view to minimizing uncertainties in terms of quantification which are strongly dependent on matrix effects.

Imaging is often used as a first step in SCO because it is an essential contribution to the modelling, interpretation and reduction of uncertainties in radiological (gamma and neutron) measurements. In addition to assessing the dimensional characteristics of the waste block, the quality of its containment, the absence of prohibited or regulated waste; it allows us to characterize the internal structures of the matrix in terms of the localization, homogeneity and density distribution of constitutive materials. For example, in the framework of an alpha activity measurement by non-destructive methods performed on packages of $220 \mathrm{~L}$, an X-ray imaging is carried out successively over the entire package, followed by gamma spectrometric and neutron measurements. High-energy photon imaging provides information about the density of the materials and the filling level of the package, information used in the numerical model (see Fig. 27) to refine the calculation of radiation attenuation corrections in the package, and thereby reduce the uncertainties in gamma spectrometric and neutron measurements. Moreover, gamma spectrometry can measure the isotopic composition of plutonium. In combination with the neutron measurement, the contribution of each plutonium isotope is thus determined.

As part of a destructive coring expertise on concrete packages, it is also possible to use high-energy photon imaging to target coring areas (outer envelope or waste) when the internal structure of the package is not known 

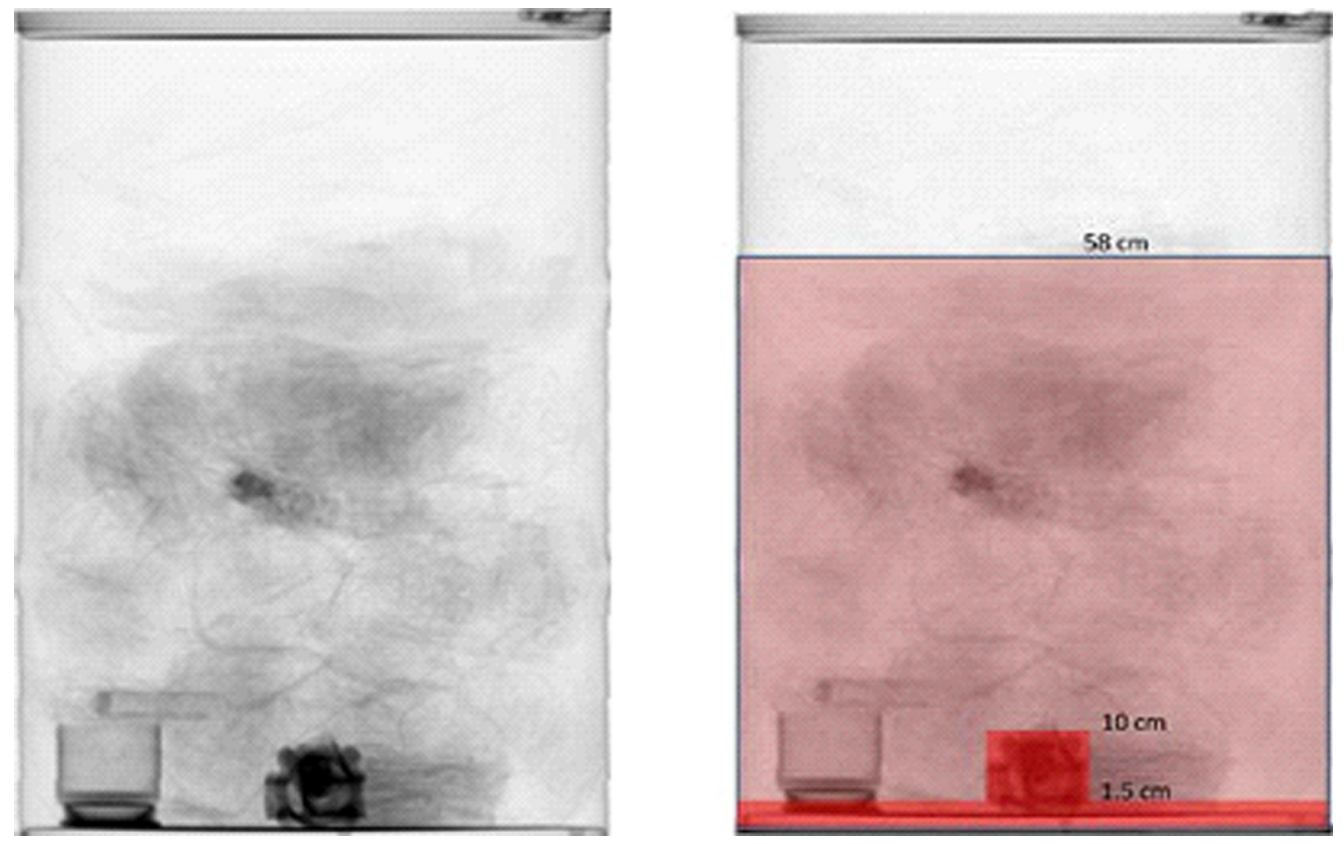

Fig. 27. X-ray of a $220 \mathrm{~L}$ waste package (left) and geometry used for Monte Carlo modelling (at right in red, a homogeneous matrix of light waste up to $58 \mathrm{~cm}$ filling height, and dense metallic objects at the bottom of the drum).

870-liter waste package

$\varnothing 100 \mathrm{~cm}-1.8$ tons
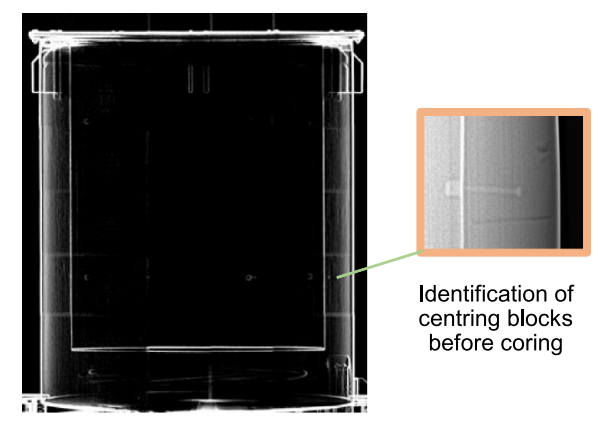

Identification of centring blocks before coring

Fig. 28. Example of high-energy photon radiography exhibiting centring studs, performed before destructive testing to determine coring positions.

with sufficient precision, in order to avoid objects that could damage the corer (such as centring pins or metallic objects, as shown in Fig. 28).

Finally, prior to radiochemical analyses, it may be necessary to carry out gamma spectrometry on samples taken from the package, in order to determine their activity, to optimise the analysis protocol, under a hood or in a glove box, and to check the homogeneity of the samples.

The development of new characterization methods is continuously under progress, partly in collaboration with
ANDRA. Examples include:

- High-energy photon imaging with two LINAC energies that will allow access, in addition to density, to the average atomic number in a waste package;

- the development of in-situ high-energy photon imaging with a low dosimetric impact, in order to allow the characterization of historic packages on their original storage site;

- Photofission for the assessment of the mass of fissile material present in dense and voluminous packages;

- the Cavity Ring Down Spectroscopy (CRDS) technique for the measurement of tritium emitted during the degassing of a waste package;

- Accelerator Mass Spectrometry by (AMS), a particle acceleration measurement method developed as an alternative to liquid scintillation for the measurement of long-lived radionuclides ${ }^{36} \mathrm{Cl},{ }^{129} \mathrm{I},{ }^{41} \mathrm{Ca}$, and ${ }^{10} \mathrm{Be}$ with low levels of radioactivity.

Finally, the developments of high-energy photon imaging and photofission are associated with an increase in experimental capacities with the recent replacement, at CEA Cadarache, of an historic ${ }^{60} \mathrm{Co}$ tomography unit called TRANSEC by the CINPHONIE irradiation cell, currently equipped with a $9 \mathrm{MeV}$ electron LINAC. This replacement allowed a substantial gain over the measurement time which has thus passed from a few hours to a few minutes. Current studies aim to use a more energetic and more intense beam produced by a $20 \mathrm{MeV}$ LINAC to examine large volume and dense packages. 


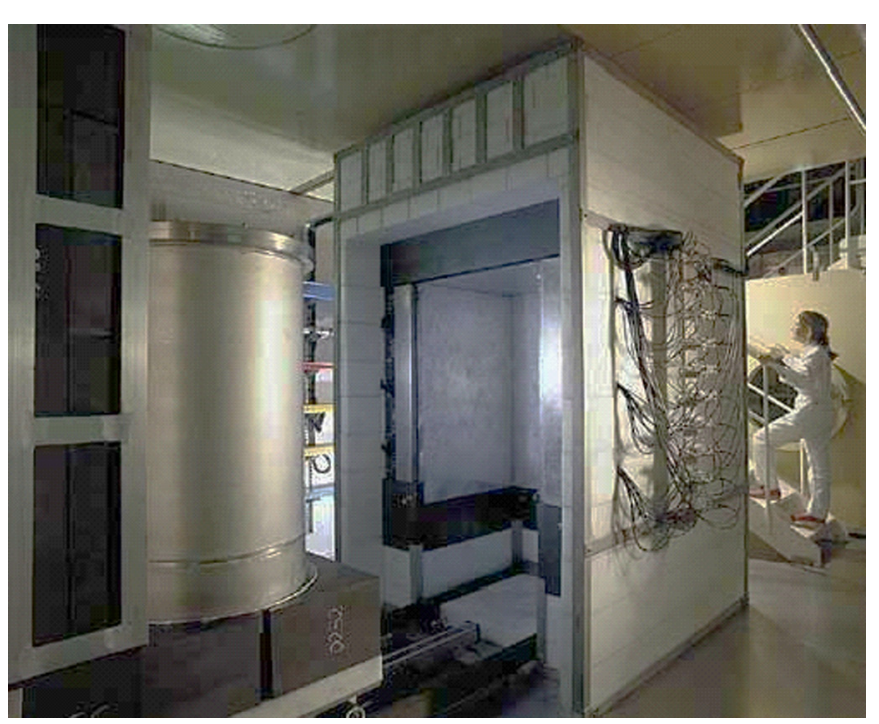

Fig. 29. CHICADE's graphite neutron measurement cell for $200 \mathrm{~L}$ drums SYMETRIC, including $99{ }^{3}$ He proportional counters with charge preamplifiers connected to an electronics counting coincidences between spontaneous fission neutrons (passive mode) or prompt and delayed fission neutrons induced by the use of a $2 \cdot 10^{9}$ n.s ${ }^{-1}$ high flux neutron generator (active neutron interrogation).

\section{The CHICADE facility}

The basic nuclear facility CHICADE, at CEA Cadarache, is an experimental platform dedicated to the characterisation of radioactive waste, from $R \& D$ to assistance in the field of their operational management.

CHICADE is a unique multi-disciplinary collaborative platform dedicated to nuclear characterisation, beginning with a sample of the order of the microgram right up to the radioactive waste packages of several tons, ranging from $\mathrm{Bq}$ to TBq.

It thus combines means of non-destructive characterisation, which include gamma spectrometry (such as the station of Fig. 3), passive and active neutron measurements in SYMETRIC equipment (Fig. 29), high energy photon imaging in CINPHONIE facility (Fig. 13) which will also be used for active photon interrogation (photofission), degassing measurement (like in Fig. 11), with means of destructive characterisation, such as sampling by coring (Fig. 30) and cutting (Fig. 23). It also deals with the measurement of physico-chemical properties (diffusion permeability, see Figs. 24 and 25), radiochemical and chemical analyses (separative and extractive chemistry, $\alpha$ and $\gamma$ spectrometry, dosage of long-lived $\beta$-emitters, see Fig. 26).

\section{Conclusion and prospects}

As demonstrated in this paper, coupling non-destructive and destructive examinations is mandatory to achieve the most comprehensive characterization of radioactive waste packages. Nuclear non-destructive assay allows determining in a limited time the global activity of the waste, by measuring gamma and neutron radiations spontaneously emitted by the radioactive and nuclear materials (passive techniques), or induced in the latter by an external source (active techniques). In addition, high-energy photon imaging allows a non-destructive 3D inspection of the internal structure of the container, possible shields, coating and waste, which enable to determine the best location for extracting representative samples in view of further chemical and radiochemical analyses. In this frame, CHICADE facility based at CEA Cadarache is a unique platform in Europe allowing such a large series of examinations including radiological nondestructive assay, gas release measurements, waste packages cutting, drilling and sampling, material behavior and containment properties testing, physical and chemical preparation of the samples in view of chemical and radiochemical analyses.

Radioactive waste characterization is in constant evolution because of the increasing demand in terms of precision and sensitivity, and technological progress in detectors, electronics, radiation sources, coring and cutting techniques, chemical and radiochemical analyses, intensive calculation means for numerical simulation and data processing, etc. The later allows simulating instruments and methods with a high reliability, so as to improve them and to get maximum information. In the coming years, photofission should be able to characterize nuclear materials in very large and dense packages and dualenergy photon imaging should provide the average atomic number of waste materials, which will greatly improve their identification together with the already available density information. In the field of neutron measurements, strategic R\&D topics are the reduction of the uncertainties due to matrix effects and radioactive product localisation, as well as the search for alternatives to ${ }^{3} \mathrm{He}$ counters. In the field of gas release measurements, Cavity Ring Down Spectroscopy (CRDS) is a promising technique to detect tritium. As far as radiochemical analyses, Accelerator Mass Spectroscopy (AMS) is a sensitive technique which would possibly supplement liquid scintillation counting to assess long-lived radionuclides with a low activity, such as ${ }^{36} \mathrm{Cl}$, ${ }^{129} \mathrm{I},{ }^{41} \mathrm{Ca}$, and ${ }^{10} \mathrm{Be}$. Moreover, necessary chemical separations often use off-line chromatographic step or liquid-liquid extraction before measurements. Those chemical treatments are tedious, time-consuming and require significant amounts of radioactive samples leading to exposure of operators to substantial doses and causes problems for waste management, such as contaminated organic solvents. The development of ion chromatography coupled to ICP-MS allows reducing chemical treatments applied to highly radioactive samples and thus contributes to the dose reduction. Another point of interest is the development of a speciation analytical process for carbon 14 in some solid wastes, with the aim of using organic and inorganic source terms for ingested carbon 14 in radiological impact studies on the releases of radioactive waste disposal facilities. 

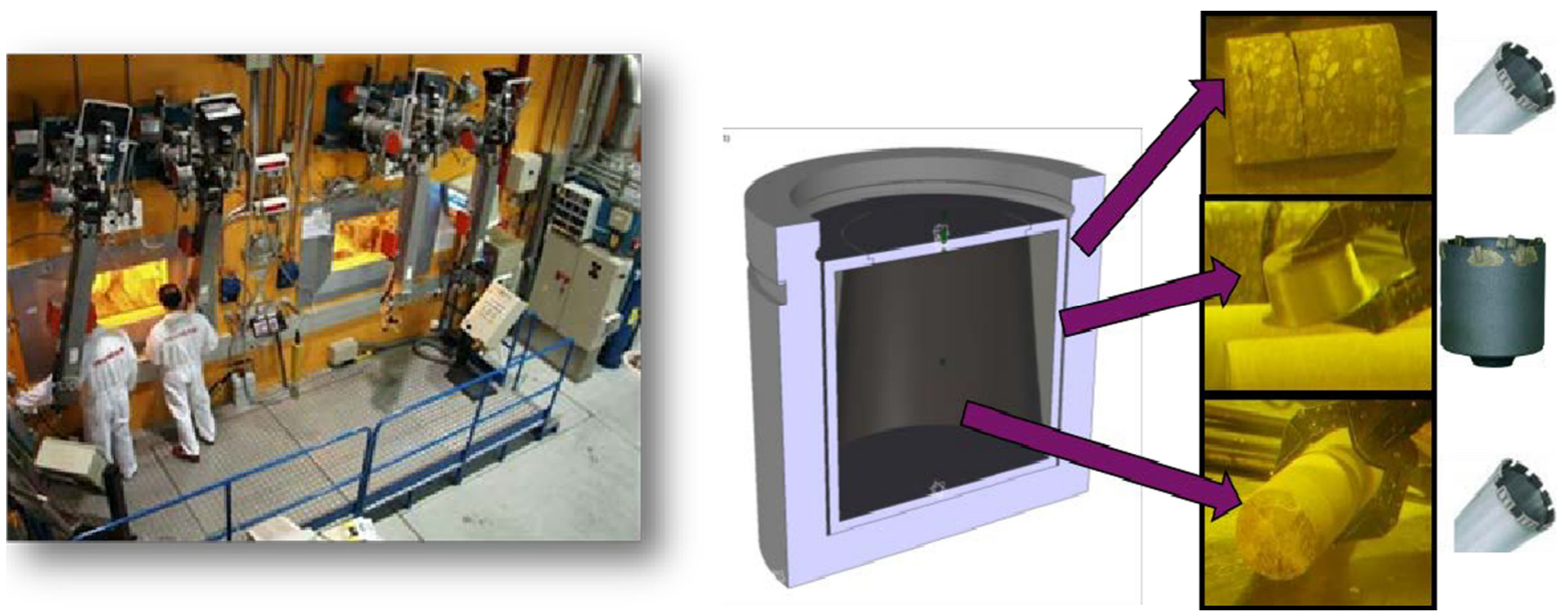

Fig. 30. The ALCESTE coring cell (at the left) and sample collection (right).

\section{References}

1. Strategy and methodology for radioactive waste characterization, IAEA-TECDOC-1537, March 2007, http://wwwpub.iaea.org/MTCD/publications/PDF/te_1537_web.pdf

2. G. Gilmore, Practical Gamma-Ray Spectrometry, 2nd edn. (Wiley, Hoboken, New Jersey, USA, 2008)

3. G.F. Knoll, Radiation Detection and Measurement, 4th edn. (Wiley, Hoboken, New Jersey, USA, 2010)

4. D. Reilly, N. Ensslin, H. Smith Jr. Passive Non Destructive Assay of Nuclear Materials Los Alamos National Laboratory, NUREG/CR-5550, $1991+2007$ addendum

5. A.-C. Simon, F. Carrel, I. Espagnon, M. Lemercier, A. Pluquet, Determination of Actinide Isotopic Composition: Performances of the IGA Code on Plutonium Spectra According to the Experimental Setup, IEEE Trans. Nucl. Sci. 58, 2 (2011)

6. F. Jallu, A. Reneleau, P. Soyer, J. Loridon, Dismantling and decommissioning: the interest of passive neutron measurement to control and characterize radioactive waste containing uranium, Nucl. Instrum. Methods Phys. Res. B 271, 48 (2012)

7. F. Jallu, P.-G. Allinei, P. Bernard, J. Loridon, D. Pouyat, L. Torreblanca, Cleaning up of a nuclear facility: destocking of $\mathrm{Pu}$ radioactive waste and nuclear Non-Destructive Assays, Nucl. Instrum. Methods Phys. Res. B 283, 15 (2012)

8. A. Leskinen, P. Fichet, M. Siitari-Kauppi, F. Goutelard, Digital autoradiography (DA) in quantification of trace level beta emitters on concrete, J. Radioanal. Nucl. Chem. 298, 153 (2013)

9. R. Haudebourg, P. Fichet, A non-destructive and on-site digital autoradiography-based tool to identify contaminating radionuclide in nuclear waste and facilities to be dismantled, J. Radioanal. Nucl. Chem. 309, 551 (2016)

10. M. Ferry, Y. Ngono-Ravache, C. Aymes-Chodur, M.C. Clochard, X. Coqueret, L. Cortella, E. Pellizzi, S. Rouif, S.
Esnouf, Ionizing radiation effects in polymers, in Reference Module in Materials Science and Materials Engineering (Elsevier, 2016)

11. N. Estre, D. Eck, J.-L. Pettier, E. Payan, C. Roure, E. Simon, High-energy X-ray imaging applied to non destructive characterisation of large nuclear waste drums, IEEE Trans. Nucl. Sci. 62, 3104 (2015)

12. A. Lyoussi et al., Method and device for analysing radioactive objects using neutrons, French patent No. WO/2000/062099 (2000)

13. H. Toubon et al., in Method for Ascertaining the Characteristics of the Radiological Content of Canister of Compacted Hull and Nozzle Waste Resulting from Reprocessing at The Hague of Irradiated Fuel Assemblies from Light Water Reactors, Safewaste Conference October 2-4 (Montpellier, 2000)

14. F. Carrel, M. Agelou, M. Gmar, F. Lainé, Detection of highenergy delayed gammas for nuclear waste package characterisation, Nucl. Instrum. Methods Phys. Res. A 652, 137 (2011)

15. F. Carrel, M. Agelou, M. Gmar, F. Lainé, J. Loridon, J.-L. Ma, C. Passard, B. Poumarède, Identification and differentiation of actinides inside nuclear waste packages by measurement of delayed gammas, IEEE Trans. Nucl. Sci. 57, 2862 (2010)

16. E. Simon, B. Perot, F. Jallu, S. Plumeri, Feasibility study of fissile mass quantification by photofission delayed gamma rays in radioactive waste packages using MCNPX, Nucl. Instrum. Methods Phys. Res. A 840, 28 (2016)

17. J.-L. Ma, C. Carasco, B. Perot, E. Mauerhofer, J. Kettler, A. Havenith, Prompt gamma neutron activation analysis of toxic elements in radioactive waste packages, Appl. Radiat. Isot. 70, 1261 (2012)

18. T. Nicol, C. Carasco, B. Perot, J.L. Ma, E. Payan, E. Maeurhofer, Quantitative comparison between PGNAA measurements and MCNPX simulations, J. Radioanal. Nucl. Chem. 306, 1 (2015) 
19 .

Nicol, B. Pérot, C. Carasco, E. Brackx, A. Mariani, C. Passard, E. Mauerhofer, J. Collot, Feasibility study of $235 \mathrm{U}$ and $239 \mathrm{Pu}$ characterization in radioactive waste drums using neutroninduced fission delayed gamma rays, Nucl. Instrum. Methods Phys. Res. A 832, 85 (2016)
20. T. Wattez, A. Duhart, S. Lorente, Modeling of nuclear species diffusion through cement-based materials, Transp. porous media 98, 699 (2013)

Cite this article as: Bertrand Pérot, Fanny Jallu, Christian Passard, Olivier Gueton, Pierre-Guy Allinei, Laurent Loubet, Nicolas Estre, Eric Simon, Cédric Carasco, Christophe Roure, Lionel Boucher, Hervé Lamotte, Jérôme Comte, Maïté Bertaux, Abdallah Lyoussi, Pascal Fichet, Frédérick Carrel, The characterization of radioactive waste: a critical review of techniques implemented or under development at CEA, France, EPJ Nuclear Sci. Technol. 4, 3 (2018) 